\title{
Hypoxia Inducible Factor-1a Overexpression Enhances the Efficacy of Mesenchymal Stem Cells Derived Extracellular Vesicles for Cardiac Repair After Myocardial Infarction
}

\section{Qingjie Wang}

Changzhou No.2 People's Hospital

\section{Le Zhang}

Wuxi Children's Hospital

\section{Zhiqin Sun}

Changzhou City No.2 People's Hospital

\section{Boyu Chi}

Changzhou City No.2 People's Hospital

\section{Ailin Zou}

Changzhou City No.2 People's Hospital

\section{Lipeng Mao}

Changzhou City NO.2 People's Hospital

\section{Xu Xiong}

Changzhou City No.2 People's Hospital

JianGuang Jiang

Changzhou City No.2 People's Hospital

Ling Sun ( $\square$ sunling85125@hotmail.com )

Department of cardiology https://orcid.org/0000-0001-6444-9470

\section{Wenwu Zhu}

Xuzhou Central Hospital

Yuan Ji

Changzhou City No.2 People's Hospital

\section{Research}

Keywords: extracellular vesicles (EVs), cardioprotection, mesenchymal stem cells (MSCs), endothelial cells

Posted Date: August 6th, 2021 
DOl: https://doi.org/10.21203/rs.3.rs-774289/v1

License: (c) (1) This work is licensed under a Creative Commons Attribution 4.0 International License. Read Full License 


\section{Abstract}

\section{Aims}

Naturally secreted extracellular vesicles (EVs) play important roles in stem-mediated cardioprotection. This study aimed to investigate the cardioprotective function and underlying mechanisms of EVs derived from HIF-1a engineered mesenchymal stem cells (MSCs) in a rat model of AMI.

\section{Methods and Results}

EVs isolated from HIF-1a engineered MSCs (HIF-1a-EVs) and control MSCs (MSCs-EVs) were prepared. In in vitro experiments, the EVs were incubated with cardiomyocytes and endothelial cells exposed to hypoxia and serum deprivation (H/SD); in in vivo experiments, the EVs were injected in the acutely infarcted hearts of Sprague-Dawley rats. Compared with MSCs-EVs, HIF-1a-EVs significantly inhibited the apoptosis of cardiomyocytes and enhanced angiogenesis of endothelial cells; meanwhile, HIF-1a-EVs also significantly shrunk fibrotic area and strengthened cardiac function in infarcted rats. After treatment with EVs/RGD-biotin hydrogels, we observed longer retention, higher stability in HIF-1a-EVs, and stronger cardiac function in the rats. Quantitative real-time PCR (qRT-PCR) displayed that miRNA-221-3p was highly expressed in HIF-1a-EVs. After miR-221-3p was inhibited in HIF-1a-EVs, the biological effects of HIF-1a EVs on apoptosis and angiogenesis were attenuated.

\section{Conclusion}

EVs released by MSCs with HIF-1a overexpression can promote the angiogenesis of endothelial cells and the apoptosis of cardiomyocytes via upregulating the expression of miR-221-3p. RGD hydrogels can enhance the therapeutic efficacy of HIF-1a engineered MSC-derived EVs.

\section{Introduction}

The negligible regenerative ability of cardiomyocytes limits the clinical efficacy for congestive heart failure (CHF)[1]. Apoptosis of myocardial cells are the molecular and cellular basis of progressive heart failure. Accumulating evidence shows that mesenchymal stem cells (MSCs) transplantation can promote tissue repair, regulate immunity and promote angiogenesis after myocardial infarction. MSCs function through releasing paracrine, especially extracellular vehicles (EVs)[2].

EVs are vesicles with a diameter of 50-200 nm[3], responsible for the communication between cells and cells. Moreover, MSC-derived EVs (MSC-EVs) have been shown to produce reparative effects on a variety of diseases without side-effects, including immunogenicity, tumorigenicity and teratoma formation[4]. MSC-EVs can reduce cardiac ischemia-reperfusion injury and protect heart function in ischemic heart disease.

Our previous studies demonstrated that hypoxia-conditioned hMSCs-derived exosomes protected cardiomyocyte from apoptosis. Moreover, four weeks after intramyocardial injection of MSCs EVs, the 
infarct size and number of apoptotic cells in the infarct border area were significantly reduced, and the cardiac function was improved. Hypoxia inducible factor-1a (HIF-1a) regulates cells' adaptation to hypoxia through activating a variety of angiogenic factors. In this study, we reported an efficient method for the detection of HIF-1a engineered MSCs-EVs and analyzed their molecular mechanism.

Moreover, the application of MSC-EVs in tissue repair is restricted by its low stability and short retention. EVs functions rely on their concentrations, administration routes, retention and target tissues. RGD (ArgGly-Asp) peptides can bind to integrins on the membrane of EVs. Therefore, we invented RGD hydrogels to load more EV integrins to improve the reparative ability of EVs for cardiac tissues.

\section{Methods}

\section{Humanumbilical cord MSCs Culture and Lentiviral Transduction}

Human umbilical cord MSCs were obtained from healthy donors $(n=3$, ages 20-25yrs) and cultured in aMEM with $10 \%$ FBS and $100 \mathrm{U} / \mathrm{mL}$ penicillin-streptomycin (Gibco). MSCs in passages 3-6 were used for experiments. MSCs were incubated at $37^{\circ} \mathrm{C}$ in humidified air with $5 \% \mathrm{CO}_{2}$.

MSCs were transduced with a HIF-1a-overexpressing lentivirus (Ubi-MCS-3FLAG-SV40-EGFP-IRESpuromycin) or a control lentivirus, named as HIF-1a-MSCs or NC-MSCs, respectively (Genechem Co. Ltd., Shanghai, China). $72 \mathrm{~h}$ after transduction, these cells were further confirmed by western blot analysis and fluorescence microscope.

\section{Culture of neonatal rat cardiomyocytes and human umbilical vein endothelial cells}

One-day-old (P1) S/D rats were prepared for isolation of primary cardiomyocytes. Heart ventricles were removed from neonatal pups, minced into $1-\mathrm{mm}^{3}$ pieces, then digested with a solution containing $0.25 \%$ trypsin and $0.1 \%$ collagenase II. The dissociated cells were pre-plated at $37^{\circ} \mathrm{C}$ for $1.5-2 \mathrm{~h}$. Afterwards, cardiomyocytes were separated from fibroblasts through differential adhesion, followed by culture with Dulbecco's modified Eagle's medium (DMEM; Gibco, New York, USA) containing 5\% fetal bovine serum (FBS, Gibco, New York, USA) and $10 \%$ horse serum (HS, Gibco, New York, USA) at $37^{\circ} \mathrm{C}$ in $5 \% \mathrm{CO}_{2}$ air.

Human umbilical vein endothelial cells (HUVECs) were cultured in Dulbecco's modified Eagle medium (DMEM, Gibco, USA) mixed with $10 \%$ fetal bovine serum (FBS, Gibco, USA), $100 \mathrm{U} / \mathrm{mL}$ penicillin, $100 \mathrm{mg} / \mathrm{mL}$ streptomycin and $110 \mathrm{mg} / \mathrm{mL}$ sodium pyruvate at $37^{\circ} \mathrm{C}$ in $5 \% \mathrm{CO}_{2}$ air.

\section{Western Blot}

Bradford assay (5000202; BioRad, Hercules, CA, USA) was carried out to quantify cell lysates. Western blotting was performed using antibodies including HIF-1a (36169; Cell Signaling Technology), antiglyceraldehyde-3-phosphate dehydrogenase (GAPDH) (5174; Cell Signaling Technology), TSG101 (14497, Proteintech, United States), CD63 (25682, Proteintech) and CD81 (66866, Proteintech). 


\section{Isolation of EVs}

MSCs-EVs were prepared through differential ultracentrifugation, allowed to grow in the EV-free medium to a $70-80 \%$ confluency for $48 \mathrm{~h}$. Cell debris was eliminated from the conditioned medium through centrifugation at $300 \mathrm{~g}$ for $10 \mathrm{~min}$, at $2000 \mathrm{~g}$ for $10 \mathrm{~min}$, and at $10,000 \mathrm{~g}$ for $30 \mathrm{~min}$. Then the EVs were filtrated with a $0.22 \mathrm{~mm}$ filter and harvested through ultracentrifugation at $100,000 \mathrm{~g}$ for $70 \mathrm{~min}$ at $4{ }^{\circ} \mathrm{C}$. Having been washed and resuspended in PBS, the EVs were ultracentrifuged at $100,000 \mathrm{~g}$ for $2 \mathrm{~h}$ to eliminate the contaminated proteins. After this treatment, the EVs were resuspended in PBS and stored at $-80^{\circ} \mathrm{C}$. The content of protein in the EVs was measured through a BCA protein assay kit (Promega, Madison, WI, USA). The structure of EVs was exhibited by transmission electron microscope (TEM), and their size by nanoparticle tracking analysis (NTA).

\section{Internalization of EVs}

The EVs were labeled by DiR (Beyotime, China). The H9c2 cells and HUVECs were seeded and co-cultured with DiR-labeled EVs at different concentrations. After $24 \mathrm{~h}$, the cells were washed with PBS and observed using IVIS Lumina Imaging Systems. The density of BLI signals was measured as average radiance from regions of interest (ROIs).

Moreover, the EVs were also labeled by DiL (Beyotime, China) and co-cultured with receptor cells at 2 time points $(2 \mathrm{~h}$ and $24 \mathrm{~h}$ ). Then the cells were washed with PBS and observed under fluorescence microscope.

\section{Calcein-AM and PI staining}

To assess the effects of EVs on the survival of $\mathrm{H} 9 \mathrm{C} 2$ cells, EVs were co-cultured with $\mathrm{H} 9 \mathrm{c} 2$ cells for $24 \mathrm{~h}$ under $\mathrm{H} / \mathrm{SD}$ condition and then stained with DAPI and PI, followed by fluorescence microscopy to observe the stains of red (PI) and blue (DAPI) fluorescence.

\section{Apoptosis assays}

Apoptosis was evaluated by TUNEL based on TUNEL Apoptosis Detection kit (Yisheng, Shanghai, China). Samples were mounted with mounting medium containing 4', 6'-diamidino-2- phenylindole (DAPI; Vector Laboratories). The stained nuclei were counted with a Zeiss LSM510 META microscope. The percentage of apoptotic nuclei =the total number of TUNEL-stained nuclei divided/the total number of DAPI-positive nuclei. Apoptosis was also evaluated with caspase-3/7 cell apoptosis detection kit (Ribo, China) according to the manufacturer's instructions. Caspase-3/7 green fluorescence, PI red fluorescence and hoechst33342 blue fluorescence could distinguish apoptotic cells, dead cells and living cells, respectively[5, 6].

\section{HUVECs tube formation assay}


To assess their angiogenic ability of EVs derived from MSCs, HUVECs were seeded (10,000 cells/well) in 96-well plates coated with growth-factor reduced Matrigel (356230; BD Biosciences, San Jose, CA, USA). Having been challenged with EVs or PBS for $8 \mathrm{~h}$, photos of capillary-like tube formation were captured. Tube length and number of branches were measured with ImageJ software.

\section{Animal experiments}

All procedures were approved by the Animal Care and Use Committee in Nanjing Medical University. Sprague-Dawley rats (male, 8 weeks old) were subjected to AMI model after left anterior descending (LAD) coronary ligation as previously described[7]. MSCs-EVs (100 mg) suspended in PBS or RGD hydrogels $(100 \mathrm{~mL})$ was intramyocardially injected into two sites in the border area of infarcted hearts. Rats were euthanized by administration of pentobarbital at day 3 or day 28 after Ml.

\section{Echocardiography studies}

At 28 days after EVs therapy, cardiac function was evaluated through transthoracic echocardiography (Ultramark 9; Soma Technology, Bloomfield, CT, USA), and dimensions calculated using MatLab R2011b software (MathWorks, Natick, MA, USA).

\section{Masson's trichrome and Haematoxylin-Eosin staining}

Paraffin-embedded slides were stained by Masson's trichrome. Infarcted area was measured as the percentage of fibrotic area in the total left ventricular area. The thickness of infarcted cardiac wall was calculated as the mean of three equidistant measurements on each section. Fluorescence microscopy (Leica Microsystems, Wetzlar, Germany) was used to catch images and ImageJ software (NIH) to analyze them. The inflammation was degreed using Haematoxylin-Eosin (HE) staining.

\section{Tissue apoptosis analysis}

Apoptosis was assessed with TUNEL assay (C10625; Thermo Fisher Scientific) in vivo according to related instructions.

\section{Immunofluorescence}

Immunostaining was performed with the primary antibodies including anti-a-sarcomeric actin (a-SA, a7811, Sigma-Aldrich); anti-a-smooth muscle actin (ab5694, Abcam); anti-CD31 (ab7388; Abcam, Cambridge, United Kingdom); anti-connexin43 (3512; Cell Signaling Technology); wheat germ agglutinin (WGA, W11261, Thermofisher Scientific, USA). DAPI was used for nuclear counterstaining.

\section{Injectable hydrogels}

In the mixture of Biotin-GFFYGRGD (1.14 mg) with PBS buffer $(1 \mathrm{~mL})$, the $\mathrm{pH}$ value was adjusted to 7.4 through adding $2 \mu \mathrm{L}$ of $1 \mathrm{M} \mathrm{Na}_{2} \mathrm{CO}_{3}$. Next, the compound in the suspension was completely dissolved at 
$95^{\circ} \mathrm{C}$, then cooled (as low as room temperature) till hydrogels formed. Only $100 \mu \mathrm{M}$ hydrogel was chosen for animal experiments. Biotin-GFFYGRGD was observed under scanning electron microscope (SEM).

\section{In vivo tracking of EVs}

In vivo, rats were LAD-ligated. EVs were stained with DIR, encapsulated in RGD hydrogels (EV/RGDbiotin), intramyocardially injected into two sites in the border area of infarcted hearts, and tracked by Gluc signals (IVIS Lumina Imaging Systems, Xenogen Corporation) at the indicated time points. The signal density of bioluminescence imaging (BLI) was measured from ROls.

\section{Real-Time PCR}

Exosomal RNA was extracted using Trizol reagent (Life Technologies, USA). To quantify the level of miR221-3p, cDNA was synthesized with miRNA First-Strand cDNA Synthesis Kit (by stem-loop) (Vazyme Biotech, China). Stem-loop qRT-PCR was accomplished with a FastStart Essential DNA Green Master (Roche, USA). The exosomal level of miR-221-3p was normalized to that of cel-miR-39 (C39) and calculated with the equation "relative gene expression= 2- $(\Delta$ Ctsample $-\Delta$ Ctcontrol $)$ ". The primers are listed in Supplementary Table 1.

\section{MicroRNA-inhibitor transfection}

HIF-1a engineered MSCs were allowed to grow to 70\%-80\% confluence, then transfected with synthetic miR-221-3p inhibitor or negative control (RiboBioهGuangzhou, China) on the system of Lipofectamine 2000 (Invitrogen, Grand Island, NY, USA). After a 6-h transfection, the cells were added into EV-free FBS containing a-MEM and kept for $48 \mathrm{~h}$. The conditioned medium was collected and EVs isolated as HIF-1aEV-Inhibitor ${ }^{\text {miR-221 }}$ and HIF-1a-EV-Inhibitor ${ }^{N C}$.

\section{Statistical Analyses}

The data were presented as mean \pm SEM. The quantification was performed and graphs generated using GraphPad Prism 7 (La Jolla, CA, USA). One-way ANOVA was implemented for comparison among multiple groups and t-test for comparison between two groups. $\mathrm{P}<0.05$ was considered statistically significant.

\section{Results}

\section{Characterization of EVs derived from HIF-1a-MSCs}

To verify the efficiency of lentiviral modification, green fluorescence was observed in both HIF-1a-MSCs or NC-MSCs (Fig.1A). Western blot showed that HIF-1a protein level significantly increased in HIF-1a-MSCs than in NC-MSCs (Fig.1B). EVs were then isolated from HIF-1a-MSCs and NC-MSCs by differential centrifugation (Fig.1C). TEM showed that the EVs from HIF-1a-EVs and NC-EVs had a shape of cup and a size of about $100 \mathrm{~nm}$ (Fig.1D). Specific exosomal markers (TSG101, CD81 and CD63) showed positivity 
in HIF-1a-EVs and NC-EVs (Fig.1E). The size and concentration of EVs were showed no between-group difference (Fig.1F).

EVs were tracked in vitro after being labeled with DIR and co-cultured with $\mathrm{H} 9 \mathrm{C} 2$ cells and HUVECs at different concentrations. After $24 \mathrm{~h}$, the calibration curves (Fig. 2A, 2B) presented a linear correlation between EVs level and fluorescent density.

Having been labelled with DiL $(10 \mu \mathrm{mol} / \mathrm{L}, 1 \mu \mathrm{L})$ for $24 \mathrm{~h}$, the EVs were co-cultured with $\mathrm{H} 9 \mathrm{C} 2$ cells and HUVECs for $6 \mathrm{~h}$ and HUVECs for $24 \mathrm{~h}$ under H/SD. As shown by confocal images, H9C2 and HUVECs took up labeled EVs in a time dependent manner (Fig. 2C, 2D).

\section{Biofunctions of HIF-1a-EVs}

To explore whether HIF-1a in MSCs regulates the biological functions of EVs, the cytoprotective and proangiogenic effects of HIF-1a-EVs were evaluated. HIF-1a-EVs and NC-EVs prevented apoptosis in H9c2s under H/SD condition (Fig.3A and Fig.3D). The percentages of viable cells in the HIF-1a-EVs and NC-EVs groups were significantly higher than that in the control group (Fig.3C). HIF-1a-EVs and NC-EVs groups also exhibited weakened caspase3 and caspase7 activity in cardiomyocytes (Fig. 3B and Fig.3E). Cardiomyocytes treated with HIF-1a-EVs and NC-EVs exhibited lower TUNEL-positive signals than the control cells (Fig.3F and Fig.3G). Next, the angiogenic ability of HUVECs was evaluated according to the status of microvessels, and more tubular structures developed in HUVECs as treated with HIF-1aEVs or NC-EVs (Fig. 3H and Fig. 3I). Altogether, HIF-1a-EVs exhibited evident anti-apoptosis effects on cardiomyocytes and proangiogenic effects on HUVECs in vitro.

\section{HIF-1a-EVs protected cardiac function in a rat MI model}

HIF-1a-EVs, NC-EVs or PBS (AMI control group) were intramyocardially injected into the border of infarcted hearts at post-MI 30 min. Distribution of Dil-labelled HIF-1a-EVs (EVs-Dil) in infarcted hearts was observed on post-MI Day 3. The area with positive EVs-PKH26 increased in the myocardium (Fig.4A). On post-MI Day 28, the HIF-1a-EVs and NC-EVs groups showed significant enhancement in LVEF and LVFS, compared to the PBS group (Fig. 4D and 4E). In addition, HIF-1a-EVs were superior to NC-EVs in improving systolic function (Fig. 4B). Furthermore, we observed lower level of inflammatory infiltration in the HIF-1a-EVs group than in other groups (Fig. 4C). Histological analysis exhibited smaller scars in HIF1a-EVs group than in PBS and NC-EVs groups (Fig. 4F). In addition, HIF-1a-EVs inhibited the elevation of IL-6 in the peri-infarct region (Fig. 4G). These results suggested that HIF-1a overexpression in MSCs enhanced the protective effects of MSC-derived EVs on infarcted hearts.

\section{HIF-1a-EVs promoted the survival and angiogenesis in cardiomyocytes}

According to TUNEL results at post-MI 4 weeks, the number of apoptotic cells was lowered in the myocardial boarder zone after EVs treatment, compared to that after PBS treatment. The number of TUNEL positive cells was the lowest in the HIF-1a-EVs group (Fig.5A and Fig.5B). As demonstrated by immunofluorescence at 4 weeks after infarction, arteriole density was higher in the HIF-1a-EVs group than 
in NC-EVs and PBS groups (Fig.5C and 5D). Capillary density showed similar changes (Fig.5C and 5E). Conx43 expression decreased in HIF-1a-EVs group (Fig.5F). HIF-1a-EVs played their reparative role through enhancing the angiogenesis and increasing cell survival.

\section{EVs/RGD-biotin hydrogels increased the retention and stability of HIF-1a-EVs}

RGD-biotin molecules were generated through covalently attaching Biotin-GFFYG. The biotin-GFFYGRGD had also a property of self-assembling (Fig.6A). SEM images of the RGD hydrogels exhibited disarranged nanofibers formed by RGD-biotin molecules (Fig. 6B). The biotin-GFFYGRGD was completely dissolved at $95^{\circ} \mathrm{C}$, till the formation of RGD hydrogels as it gradually fell to the room temperature (Fig. 6C). We also compared the properties of RGD hydrogels with those of biotin hydrogels without the integrin-targeting tripeptide RGD. Next, EVs and EVs adhered to RGD hydrogels (EV/RGD-biotin) were injected in the AMI rat model (Fig. 6D). The stability of EVs was assessed. Dense signals were measured in the heart at postinjection Day 1 in all groups, indicating successful EV engraftment. EV/RGD-biotin prolongated EV retention, compared to EVs alone (Fig. 6E).

At post-AMI Day 28, LVEF and LVFS increased in the presence of RGD-Biotin in HIF-1a-EVs group (Fig. 7A and 7C). The infiltration of inflammatory cells reduced in the presence of RGD-Biotin in HIF-1a-EVs group. Masson's trichrome staining also showed the attenuation in cardiac fibrosis (Fig. 7D and 7E).

\section{Inhibition of miR-221-3p countered the effects of EVs on apoptosis and angiogenesis}

As shown by RTPCR experiments (Fig. 8A), miRNA-221-3p was overexpressed in HIF-1a-EVs. To identify the responsible signaling pathways, miR-221-3p inhibitor and miR-221-3p inhibitor NC were transfected into HIF-1a-MSCs. Subsequently, HIF-1a-EVs-inhibitor ${ }^{\text {miR-221 }}$ and HIF-1a-EVs-inhibitor ${ }^{\mathrm{NC}}$ were incubated with cardiomyocytes and HUVECs under H/SD condition (Fig.8B). The number of apoptotic cells and the activity of caspase $3 / 7$ rose in HIF-1a-EVs-inhibitor ${ }^{\text {miR-221 }}$ group, compared with those in HIF-1a-EVsinhibitor $^{\mathrm{NC}}$ group (Fig. 8C-8F). HIF-1a-EVs-inhibitor ${ }^{\mathrm{miR}-221}$ curbed the construction of endothelial network compared with HIF-1a-EVs-inhibitor ${ }^{\mathrm{NC}}$, as shown by the data of endothelial tube length and branching points (Fig.8G-8H). Together, miR-221-3p inhibited the apoptosis of cardiomyocytes and boosted the angiogenesis in HUVECs.

\section{Discussion}

We have previously demonstrated that exosomes derived from human umbilical cord blood MSCs can inhibit the apoptosis of cardiomyocytes under H/SD condition[8]. HIF-1a, always upregulated under hypoxic condition, can mediate cellular adaptation to hypoxia and trigger angiogenesis $[9,10]$. This study is the first to demonstrate that EVs derived from HIF-1a engineered MSCs can exert anti-apoptotic and angiogenic effects in a rat model of AMI. Moreover, we invented a biocompatible, self-assembling and EVs-conjugating RGD hydrogel. The hydrogel strengthened the effects of EVs derived from HIF-1a engineered MSCs. Mechanistically, we identified miR-221-3p as a mediator of HIF-1a engineered MSCs (Fig. 9). 
MSCs may serve as therapeutic agents for ischemia-related diseases[11-14]. The cardiac benefits of MSCs come through their paracrine of EVs, soluble proteins, etc $[15,16]$. Exosomal secretion involves various environmental factors[17]. Under hypoxia, more exosomes are secreted, and HIF-1a may mediate this process[18]. In this study, we found that EVs secreted by MSCs with overexpressed HIF-1a exhibited angiogenic and anti-apoptotic function. The mechanism remains unclear; however, several strains of molecules may be responsible, including Rab proteins, SNARE complex, and ceramides[19]. HIF-1a interacts with Rab22A in the development of microvesicles, shedding light into the nature of HIF-1amediated secretion[20].

To explore the function of HIF-1a MSC derived EVs on ischemic hearts, we co-cultured EVs with HUVECs and cardiomyocytes. We found that EVs could be absorbed by both HUVECs and cardiomyocytes. As compared with MSC-EVs, HIF-1 a-EVs facilitated the development of tube-like structures and prolongated the survival of cardiomyocytes. In the rat AMI model, HIF-1a-EVs quickened the recovery of cardiac functions, reduced infarcted size and inhibited cardiomyocyte apoptosis, as compared to MSC-EVs. In addition, HIF-1a-EVs stimulated angiogenesis in the peri-infarct region.

The low stability and short retention have restricted the reparative abilities of MSC-EVs. Regarding that RGD peptides are highly attachable to integrins rich on the surface of EVs[21-23], we created RGD hydrogels to load more EVs, thus enhancing their therapeutic effects. Biotin serves as a hydrogelator with biocompatibility and close interactions with cell receptors, including proteins on EV membranes[24]. Therefore, biotin can increase the stability and retention of EVs. In this study, biotin-GFFYG was covalently attached to the N-terminal of RGD to make self-assembling RGD-biotin molecules (biotinGFFYGRGD). The EVs/RGD-biotin hydrogels fortified the cardiac function after AMI.

A study has verified the pro-survival effects of miR-221 in a cocktail of three miRNA mimics, on the engrafted stem cells[25]. However, the mechanisms remain underlying. In vitro studies showed that miR221 mimics transfection extended cardiomyocyte survival after hypoxia and reoxygenation $(H / R)$ The apoptosis of cardiomyocytes can be inhibited through miR-221[26]. miR-221 also employs Ddit4 and Tp53inp1 to initiate an anti-autophagic mechanism in protecting cardiac functions[27]. Other studies have also proved that miR-221 targets PUMA to protect cardiomyocytes[28]. Overexpression of miR-221 represses autophagy through targeting the $\mathrm{p} 27 / \mathrm{mTOR}$ pathway[29]. We have found that miR-221 expression level is higher in young MSCs than in aged MSCs in a rat model of myocardial infarction[30]. However, other molecules in HIF1a-EVs may also exert cardioprotective effects, which should be explored in future studies.

During post-MI cardiac repair, tissue regeneration is key step. Combination of HIF-1a-EVs with cardiomyocyte inducible stem cells, or EVs with cell fate reprogramming factors, is expected to achieve this goal. Moreover, the efficacy and safety of RGD-biotin hydrogels should be validated with preclinical studies.

\section{Conclusion}


EVs released by MSCs with stabilized overexpression of HIF-1a can exert angiogenic and anti-apoptotic effects through increasing the expression of miR-221-3p. RGD hydrogels can strengthen the therapeutic efficacy of HIF-1a engineered MSC-derived EVs on functional, histopathological and molecular levels.

\section{Declarations}

\section{Ethics approval and consent to participate}

Animal used in this study were treated in accordance with the ethics committee guidelines at Nanjing Medical University.

\section{Availability of data and materials}

The datasets and materials used in the study are available from the corresponding author.

\section{Author Contributions}

QW, YJ, WZ, LS contributed to the design of the study. QW, LZ, ZS, BC, AZ, LM and XX performed the experiments. QW, JY, WZ and LS contributed to the writing the manuscript. WZ and LS contributed to the material support of the study. All authors read and approved the final manuscript.

\section{Consent for publication}

Not applicable.

\section{Competing Interests}

The authors have declared that no competing interest exists.

\section{Acknowledgements and Funding}

This study was supported by grants from the National Natural Science Foundation of China (Grant No. 81901410), Young Talent Development Plan of Changzhou Health Commission (CZQM2020060) and the Major Research Plan of Changzhou Health Commission (ZD202020).

\section{References}

1. Campostrini G, Windt LM, van Meer BJ, Bellin M, Mummery CL: Cardiac Tissues From Stem Cells: New Routes to Maturation and Cardiac Regeneration. Circ Res 2021, 128:775-801.

2. Rani S, Ritter T: The Exosome - A Naturally Secreted Nanoparticle and its Application to Wound Healing. Adv Mater 2016, 28:5542-5552.

3. Toh WS, Lai RC, Zhang B, Lim SK: MSC exosome works through a protein-based mechanism of action. Biochem Soc Trans 2018, 46:843-853. 
4. Huang P, Wang L, Li Q, Tian X, Xu J, Xu J, Xiong Y, Chen G, Qian H, Jin C, et al: Atorvastatin enhances the therapeutic efficacy of mesenchymal stem cells-derived exosomes in acute myocardial infarction via up-regulating long non-coding RNA H19. Cardiovasc Res 2020, 116:353-367.

5. Xiong X, He Q, Liu J, Dai R, Zhang H, Cao Z, Liao Y, Liu B, Zhou Y, Chen J, et al: MicroRNA miR-215-5p regulates doxorubicin-induced cardiomyocyte injury by targeting ZEB2. J Cardiovasc Pharmacol 2021.

6. Killinger M, Vesela B, Prochazkova M, Matalova E, Kleparnik K: A single-cell analytical approach to quantify activated caspase $3 / 7$ during osteoblast proliferation, differentiation, and apoptosis. Anal Bioanal Chem 2021.

7. Zhu W, Sun L, Zhao P, Liu Y, Zhang J, Zhang Y, Hong Y, Zhu Y, Lu Y, Zhao W, et al: Macrophage migration inhibitory factor facilitates the therapeutic efficacy of mesenchymal stem cells derived exosomes in acute myocardial infarction through upregulating miR-133a-3p. J Nanobiotechnology 2021, 19:61.

8. Sun L, Zhu W, Zhao P, Wang Q, Fan B, Zhu Y, Lu Y, Chen Q, Zhang J, Zhang F: Long noncoding RNA UCA1 from hypoxia-conditioned hMSC-derived exosomes: a novel molecular target for cardioprotection through miR-873-5p/XIAP axis. Cell Death Dis 2020, 11:696.

9. Liu Y, Luo Q, Su Z, Xing J, Wu J, Xiang L, Huang Y, Pan H, Wu X, Zhang X, et al: Suppression of Myocardial Hypoxia-Inducible Factor-1alpha Compromises Metabolic Adaptation and Impairs Cardiac Function in Patients With Cyanotic Congenital Heart Disease During Puberty. Circulation 2021, 143:2254-2272.

10. Pan Z, Ma G, Kong L, Du G: Hypoxia-inducible factor-1: Regulatory mechanisms and drug development in stroke. Pharmacol Res 2021, 170:105742.

11. Guo Y, Peng Y, Zeng H, Chen G: Progress in Mesenchymal Stem Cell Therapy for Ischemic Stroke. Stem Cells Int 2021, 2021:9923566.

12. Tseng WC, Lee PY, Tsai MT, Chang FP, Chen NJ, Chien CT, Hung SC, Tarng DC: Hypoxic mesenchymal stem cells ameliorate acute kidney ischemia-reperfusion injury via enhancing renal tubular autophagy. Stem Cell Res Ther 2021, 12:367.

13. Razeghian-Jahromi I, Matta AG, Canitrot R, Zibaeenezhad MJ, Razmkhah M, Safari A, Nader V, Roncalli $\mathrm{J}$ : Surfing the clinical trials of mesenchymal stem cell therapy in ischemic cardiomyopathy. Stem Cell Res Ther 2021, 12:361.

14. Pathipati P, Lecuyer M, Faustino J, Strivelli J, Phinney DG, Vexler ZS: Mesenchymal Stem Cell (MSC)Derived Extracellular Vesicles Protect from Neonatal Stroke by Interacting with Microglial Cells. Neurotherapeutics 2021.

15. Gregorius J, Wang C, Stambouli O, Hussner T, Qi Y, Tertel T, Borger V, Mohamud Yusuf A, Hagemann $\mathrm{N}$, Yin D, et al: Small extracellular vesicles obtained from hypoxic mesenchymal stromal cells have unique characteristics that promote cerebral angiogenesis, brain remodeling and neurological recovery after focal cerebral ischemia in mice. Basic Res Cardio/ 2021, 116:40. 
16. Han J, Yang S, Hao X, Zhang B, Zhang H, Xin C, Hao Y: Extracellular Vesicle-Derived microRNA-410 From Mesenchymal Stem Cells Protects Against Neonatal Hypoxia-Ischemia Brain Damage Through an HDAC1-Dependent EGR2/Bcl2 Axis. Front Cell Dev Bio/ 2020, 8:579236.

17. Kang X, Jiang L, Chen X, Wang X, Gu S, Wang J, Zhu Y, Xie X, Xiao H, Zhang J: Exosomes derived from hypoxic bone marrow mesenchymal stem cells rescue OGD-induced injury in neural cells by suppressing NLRP3 inflammasome-mediated pyroptosis. Exp Cell Res 2021, 405:112635.

18. Guo Z, Wang X, Yang Y, Chen W, Zhang K, Teng B, Huang C, Zhao Q, Qiu Z: Hypoxic Tumor-Derived Exosomal Long Noncoding RNA UCA1 Promotes Angiogenesis via miR-96-5p/AMOTL2 in Pancreatic Cancer. Mol Ther Nucleic Acids 2020, 22:179-195.

19. Bobrie A, Colombo M, Raposo G, Thery C: Exosome secretion: molecular mechanisms and roles in immune responses. Traffic 2011, 12:1659-1668.

20. Wang T, Gilkes DM, Takano N, Xiang L, Luo W, Bishop CJ, Chaturvedi P, Green JJ, Semenza GL: Hypoxia-inducible factors and RAB22A mediate formation of microvesicles that stimulate breast cancer invasion and metastasis. Proc Natl Acad Sci U S A 2014, 111:E3234-3242.

21. Wang H, Cui J, Zheng Z, Shi Q, Sun T, Liu X, Huang Q, Fukuda T: Assembly of RGD-Modified Hydrogel Micromodules into Permeable Three-Dimensional Hollow Microtissues Mimicking in Vivo Tissue Structures. ACS App/ Mater Interfaces 2017, 9:41669-41679.

22. Zheng W, Wang Z, Song L, Zhao Q, Zhang J, Li D, Wang S, Han J, Zheng XL, Yang Z, Kong D: Endothelialization and patency of RGD-functionalized vascular grafts in a rabbit carotid artery model. Biomaterials 2012, 33:2880-2891.

23. Hoshino A, Costa-Silva B, Shen TL, Rodrigues G, Hashimoto A, Tesic Mark M, Molina H, Kohsaka S, Di Giannatale A, Ceder S, et al: Tumour exosome integrins determine organotropic metastasis. Nature 2015, 527:329-335.

24. Bhuniya S, Park SM, Kim BH: Biotin-amino acid conjugates: an approach toward self-assembled hydrogelation. Org Lett 2005, 7:1741-1744.

25. Hu S, Huang M, Nguyen PK, Gong Y, Li Z, Jia F, Lan F, Liu J, Nag D, Robbins RC, Wu JC: Novel microRNA prosurvival cocktail for improving engraftment and function of cardiac progenitor cell transplantation. Circulation 2011, 124:S27-34.

26. Daiber A, Andreadou I, Oelze M, Davidson SM, Hausenloy DJ: Discovery of new therapeutic redox targets for cardioprotection against ischemia/reperfusion injury and heart failure. Free Radic Biol Med 2021, 163:325-343.

27. Chen Q, Zhou Y, Richards AM, Wang P: Up-regulation of miRNA-221 inhibits hypoxia/reoxygenationinduced autophagy through the DDIT4/mTORC1 and Tp53inp1/p62 pathways. Biochem Biophys Res Commun 2016, 474:168-174.

28. Yu B, Gong M, Wang Y, Millard RW, Pasha Z, Yang Y, Ashraf M, Xu M: Cardiomyocyte protection by GATA-4 gene engineered mesenchymal stem cells is partially mediated by translocation of miR-221 in microvesicles. PLoS One 2013, 8:e73304. 
29. Su M, Wang J, Wang C, Wang X, Dong W, Qiu W, Wang Y, Zhao X, Zou Y, Song L, et al: MicroRNA-221 inhibits autophagy and promotes heart failure by modulating the p27/CDK2/mTOR axis. Cell Death Differ 2015, 22:986-999.

30. Sun L, Zhu W, Zhao P, Zhang J, Lu Y, Zhu Y, Zhao W, Liu Y, Chen Q, Zhang F: Down-Regulated Exosomal MicroRNA-221 - 3p Derived From Senescent Mesenchymal Stem Cells Impairs Heart Repair. Front Cell Dev Biol 2020, 8:263.

\section{Figures}


A

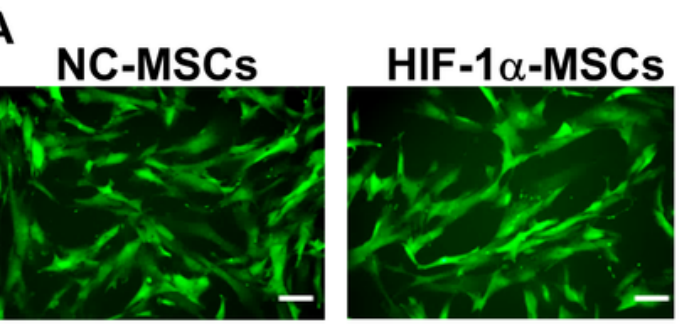

B
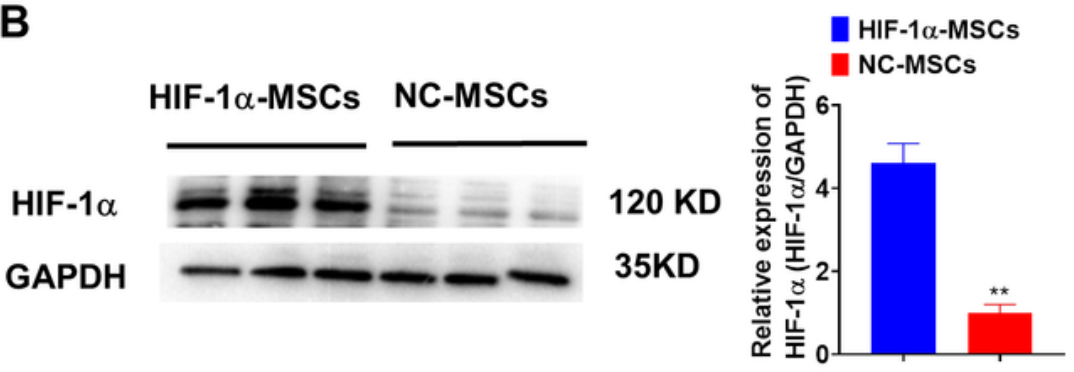

C

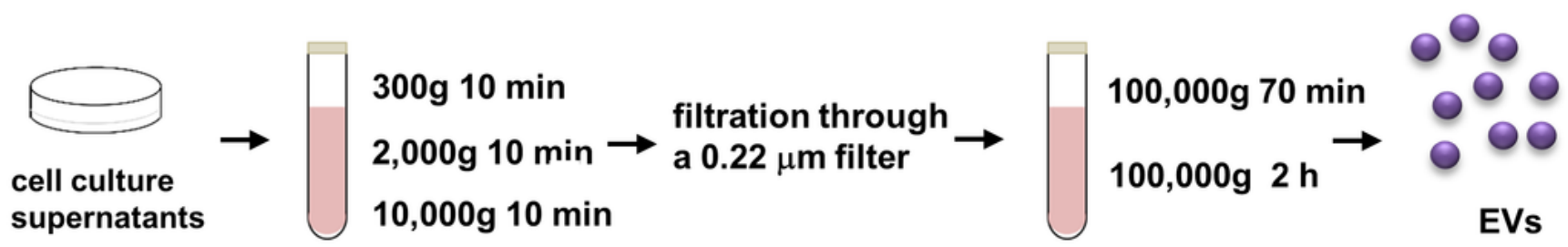

D

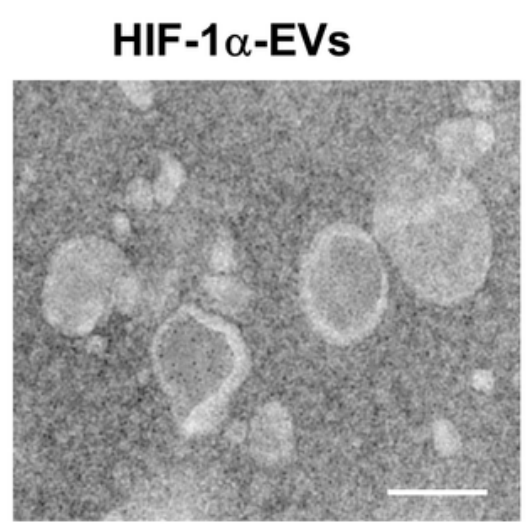

NC-EVs

E
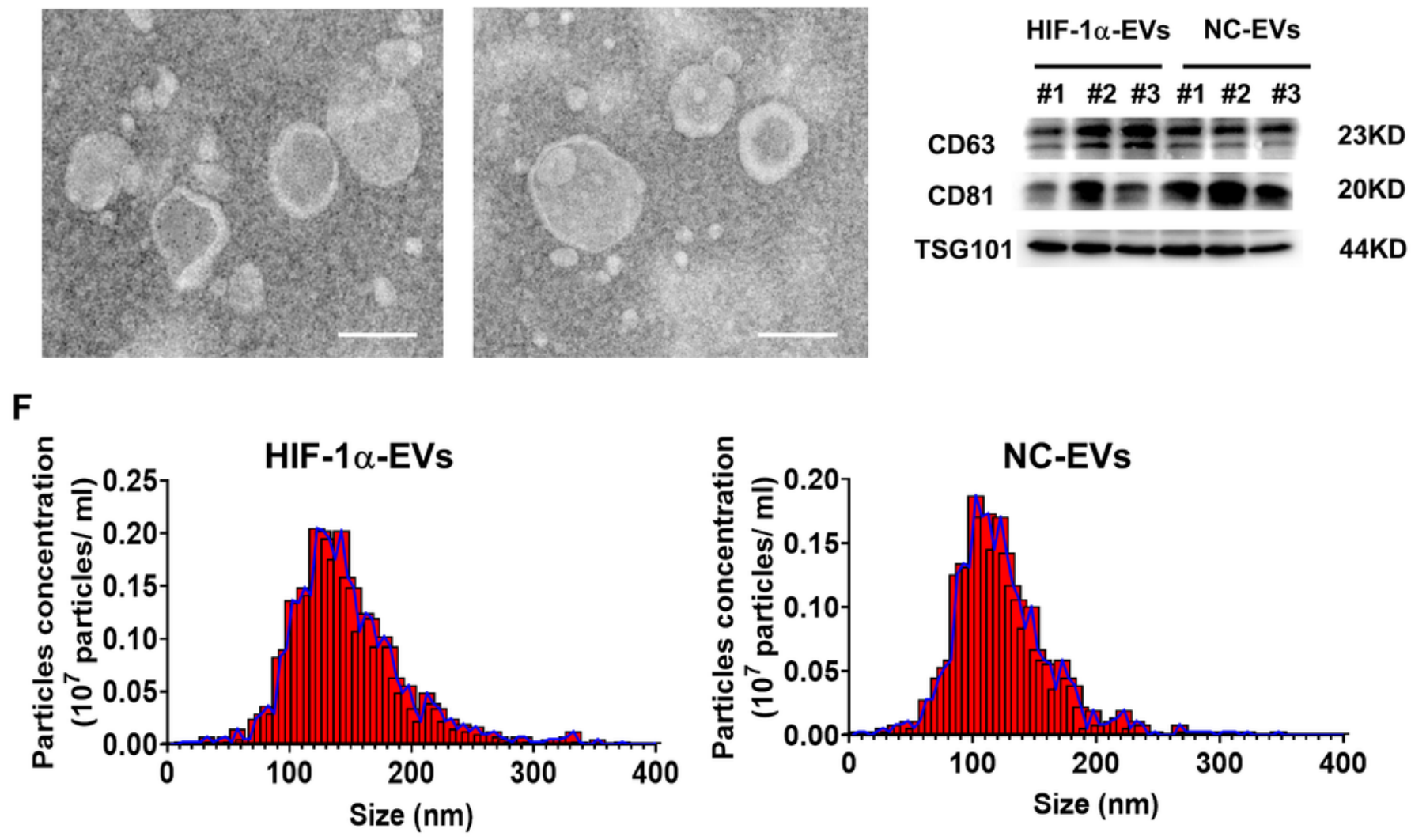

Figure 1

Lentiviral transduction, isolation and characterization of MSCs derived EVs. (A) Successful lentiviral transduction was confirmed by positive fluorescence signal under microscope. Scale bar $=100 \mu \mathrm{m} . \mathrm{n}=3$ for each group. (B) Western blot images showed HIF-1a protein levels in HIF-1a-MSCs and NC-MSCs groups. $n=3$ for each group. (C) EVs were isolated from HIF-1a-MSCs and NC-MSCs groups using a standard protocol of serial, differential centrifugation and ultracentrifugation methods. (D) TEM images 
of EVs derived from HIF-1 a-MSCs and NC-MSCs. $n=3$ for each group. Scale bar $=100 \mathrm{~nm}$ (E) The exosomal protein markers of TSG101, CD63, and CD81 in EVs isolated from HIF-1a-MSCs and NC-MSCs. $\mathrm{n}=3$ for each group. (F) The particle size distribution and particle concentration were analyzed by nanoparticle tracking analysis. $n=3$ for each group. Continuous variables were described by means \pm SEM. $* * \mathrm{P}<0.01$.

A $(\mu \mathrm{g} / \mu \mathrm{l})$
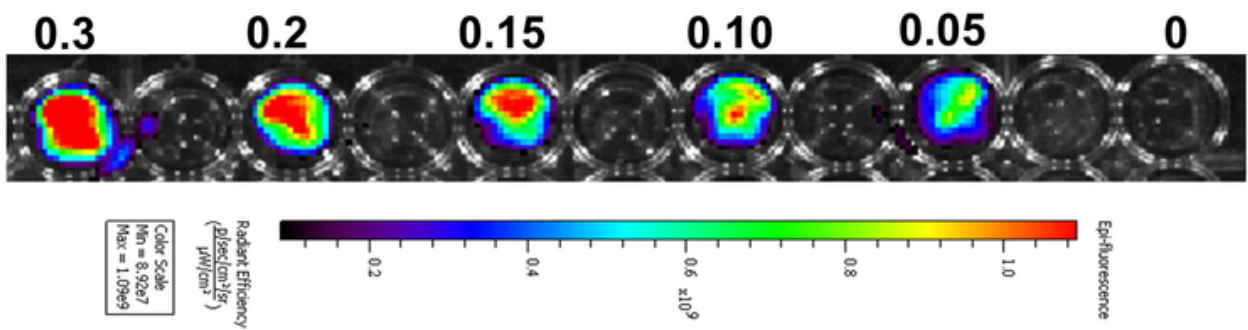

B

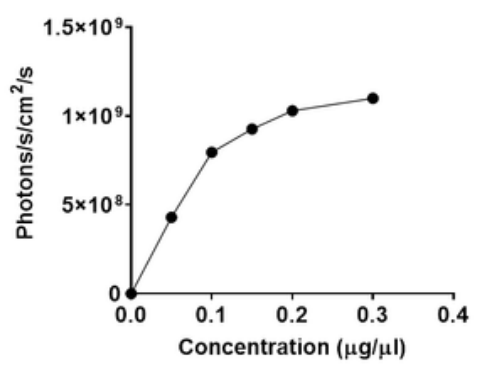

C

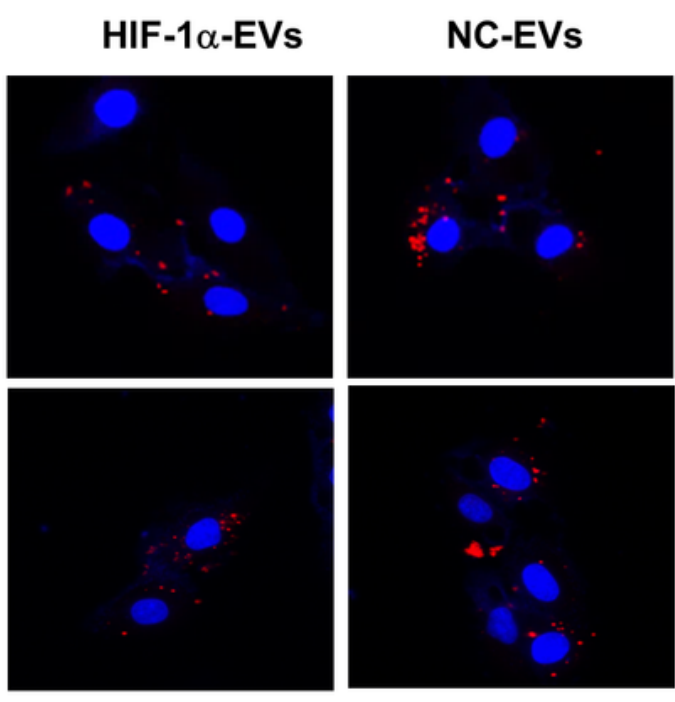

$2 \mathrm{~h}$
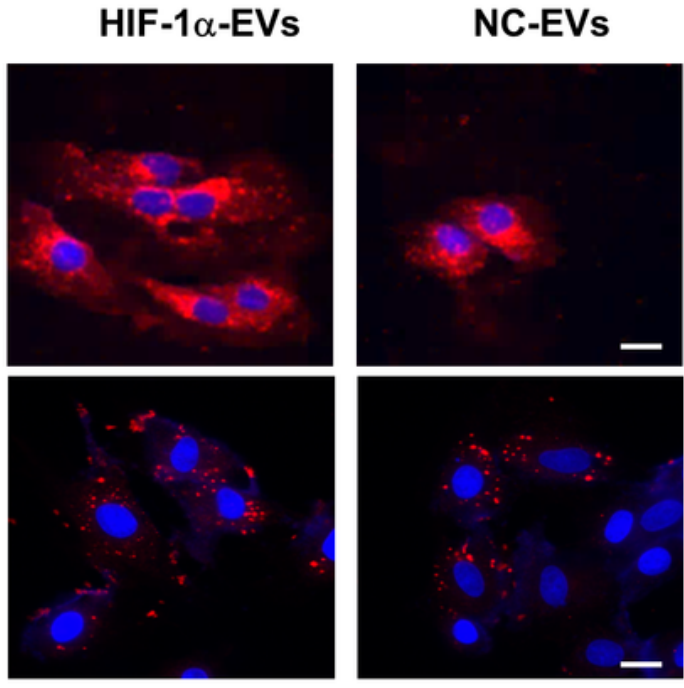

HUVECS

\section{4h}

Dil-EVs / DAPI / MERGE

Figure 2 
EVs isolated from MSCs could be absorbed by cardiomyocytes and endothelial cells in vitro. (A and B) DiR-labeled EVs with various concentrations was co-cultured with $\mathrm{H} 9 \mathrm{C} 2$ cells and internalization of EVs was detected. (C) Confocal images showed that red fluorescence of dye Dil labeled EVs were endocytosed by HUVECs and H9C2s $6 \mathrm{~h}$ and $24 \mathrm{~h}$ after incubation. Scale bar $=50 \mu \mathrm{m}$.

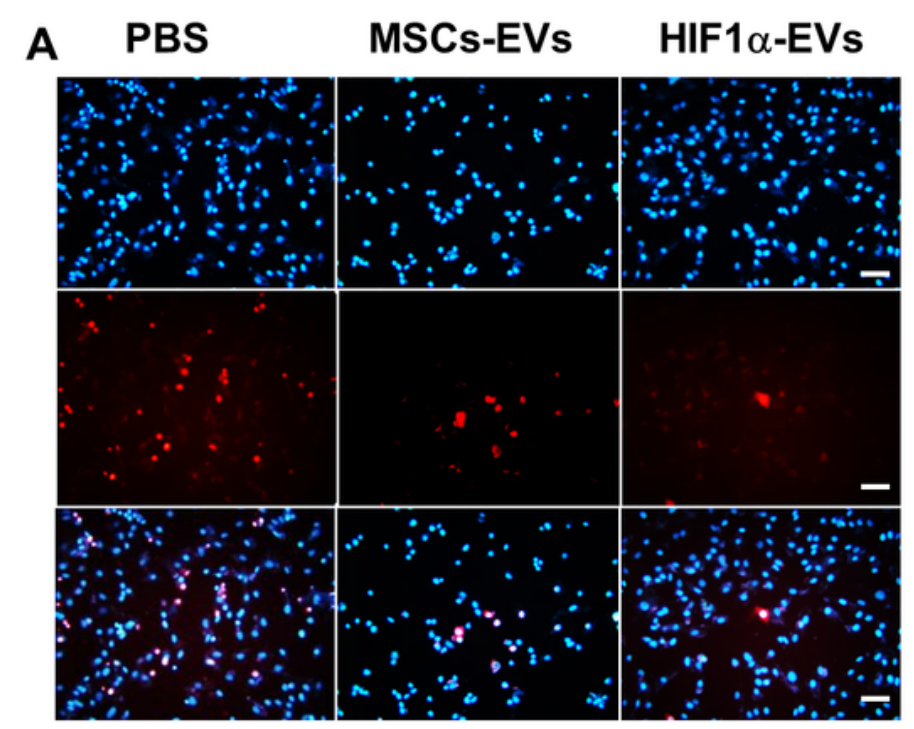

PI / Hoechest33342
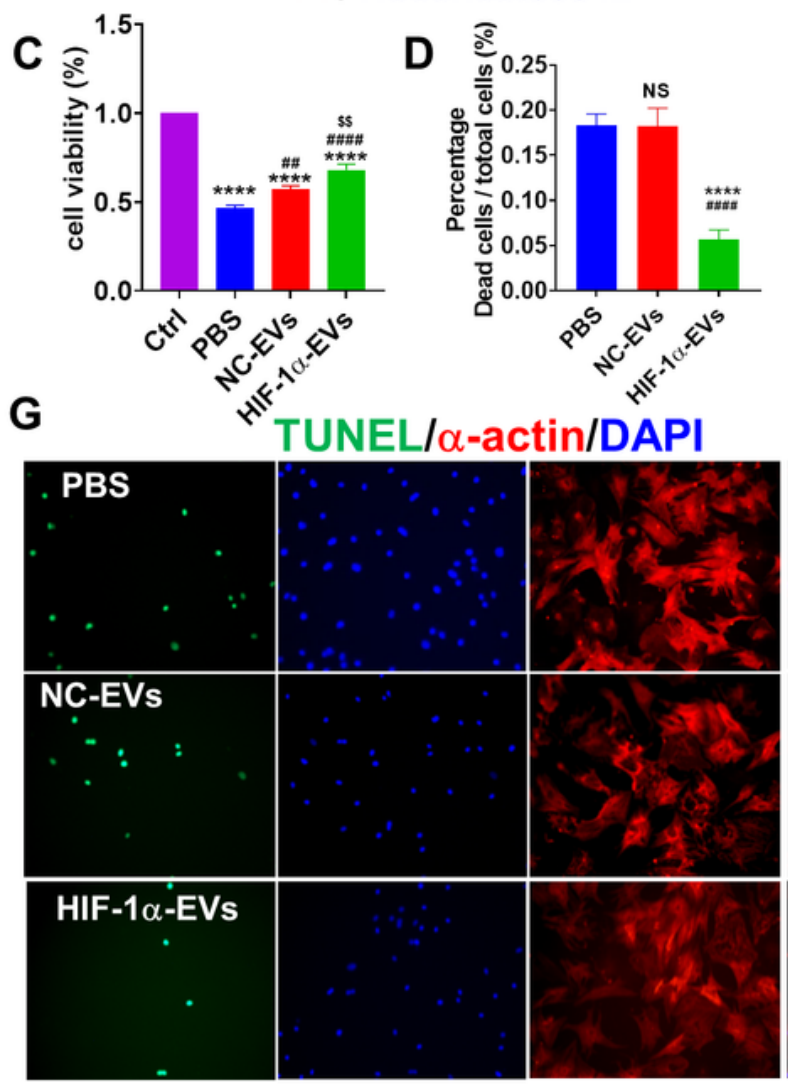

B

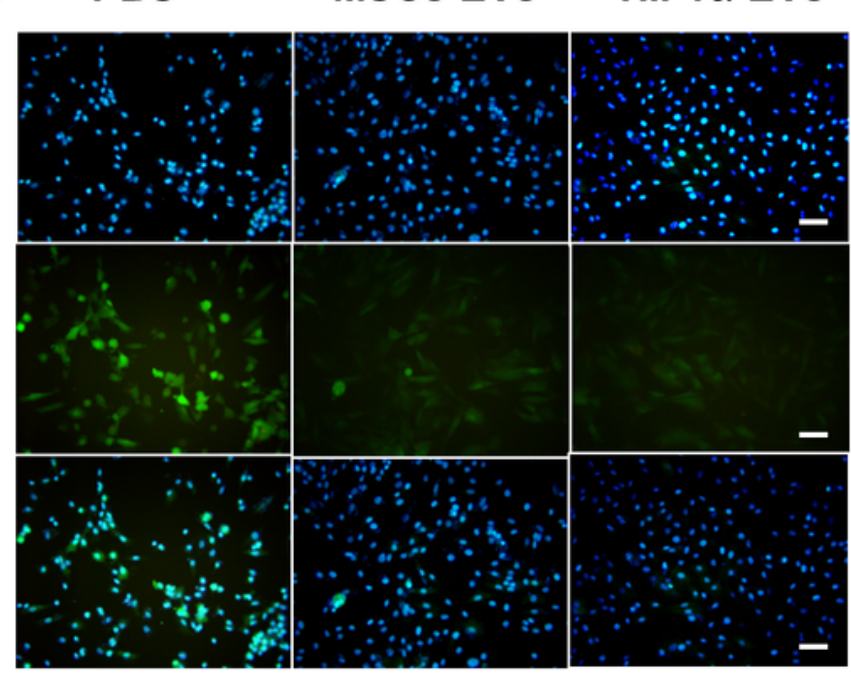

Caspase3/7 / Hoechest33342
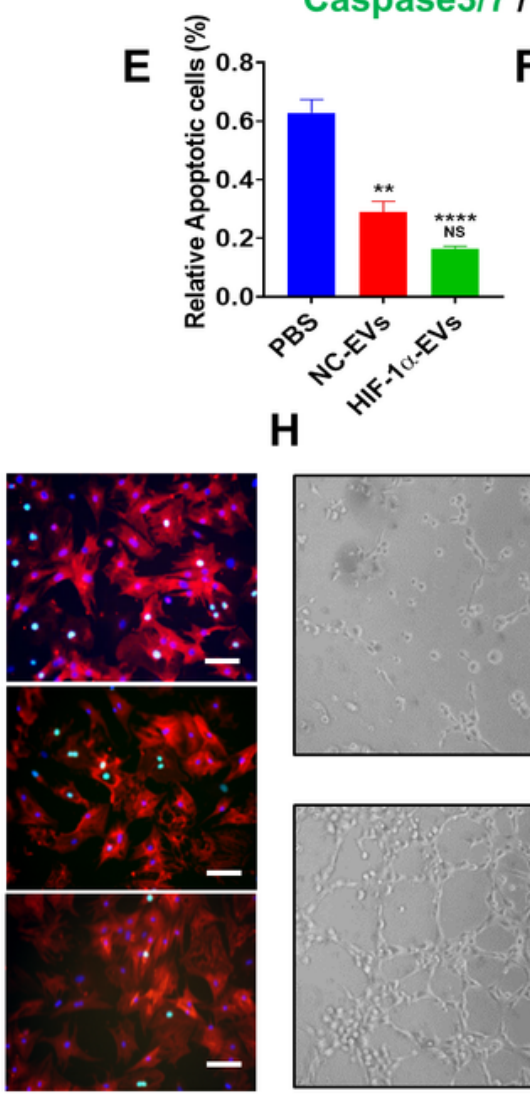

$\mathbf{F}$
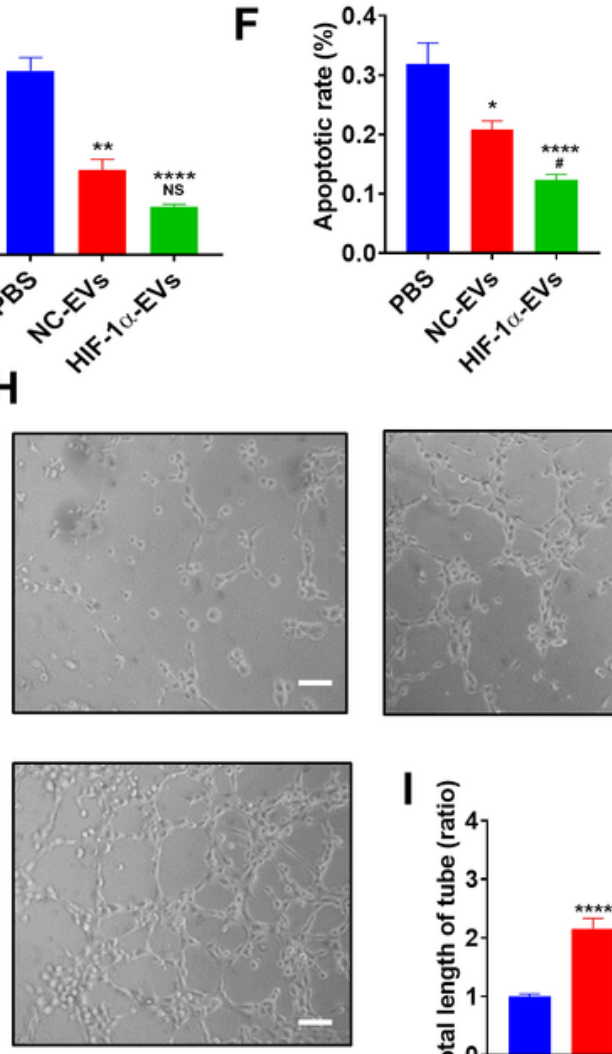
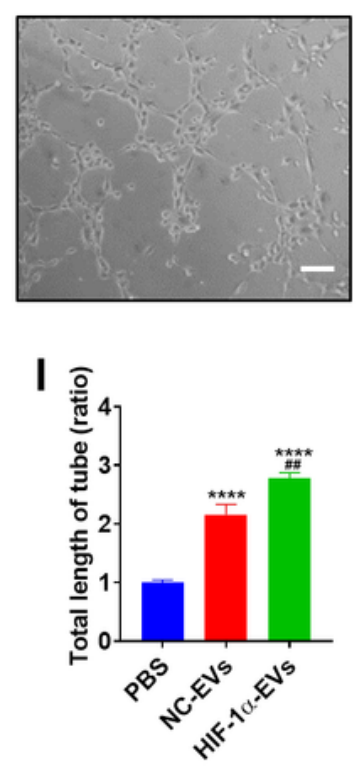

Figure 3 
HIF-1a-EVs exhibited an anti-apoptosis effects in cardiomyocytes and a proangiogenesis effects in HUVECs in vitro. (A) Hoechest33342/PI double staining was used to observe the survival of H9c2 cardiomyocytes treated with PBS, MSC-EVs or HIF-1a-EVs under H/SD condition. Scale bar $=100 \mu \mathrm{m}$. (B) Caspase3/caspase7 activity of H9c2 cardiomyocytes treated with PBS, MSC-EVs or HIF-1a-EVs under $\mathrm{H} / \mathrm{SD}$ condition. Scale bar $=100 \mu \mathrm{m}$. (C) HIF-1a EVs improved cell viability of H9c2 cardiomyocytes under $\mathrm{H} / \mathrm{SD}$ condition. (D) Quantitative analysis of viable cells treated with PBS or EVs. (E) Quantitative analysis of caspase3/caspase7 positive cells between the three groups. (F) Quantitative analysis of TUNEL-positive cells between the three groups. (G) Representative photographs showing the TUNELpositive cells in cardiomyocytes among the different groups. Green, TUNEL-positive nuclei; red, a-actin; blue, DAPI-stained nuclei. Scale bar $=100 \mu \mathrm{m}$. $(\mathrm{H})$ Representative images showing tube formation in HUVECs treated with PBS, MSC-EVs or HIF-1a-EVs. Scale bar $=100 \mu \mathrm{m}$. (I) Quantification of tube length in each group. Continuous variables were described by means $\pm S E M$. ${ }^{*} P<0.05,{ }^{*} P<0.01,{ }^{\star \star *} * P<0.0001$ vs. PBS group; $\# \mathrm{P}<0.05, \# \# \mathrm{P}<0.01$ vs. NC-EVs group; NS, not significant. 
A

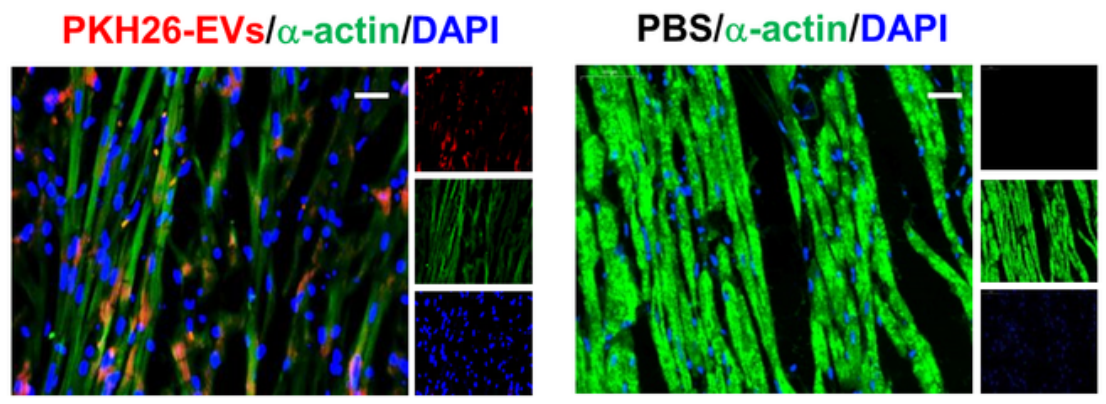

B

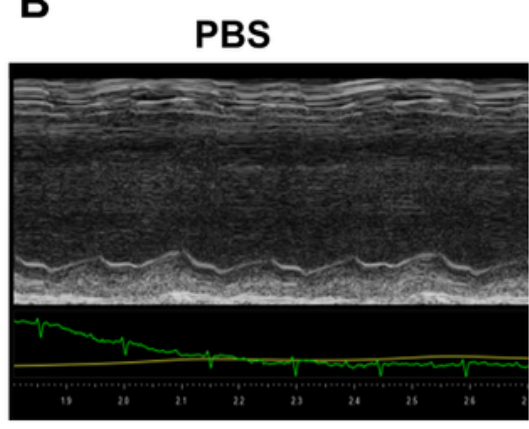

NC-EVs

HIF-1 $\alpha-E V s$

C

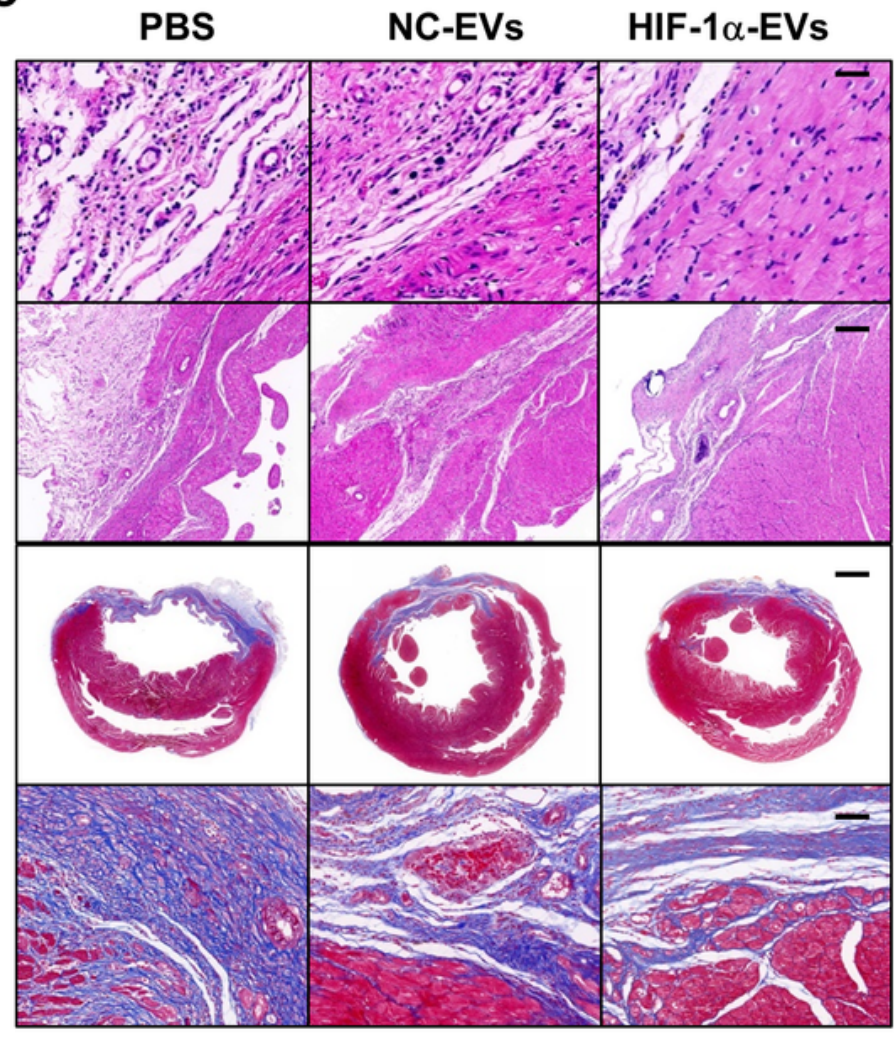

D

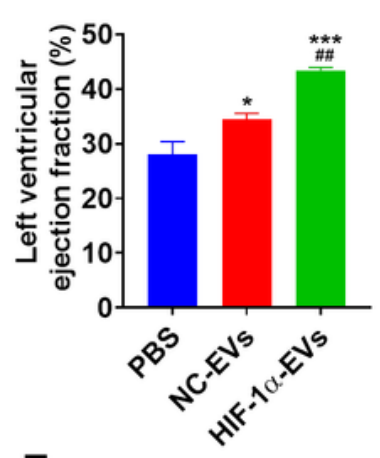

$\mathbf{F}$

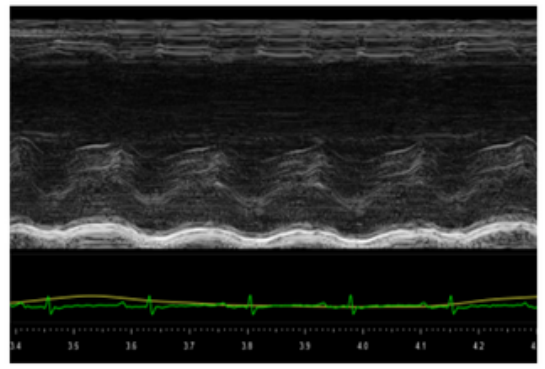

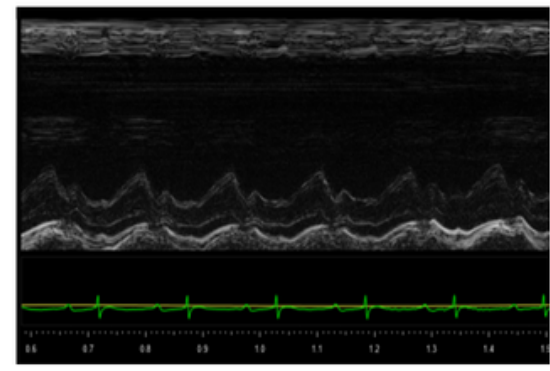

$\mathbf{E}$

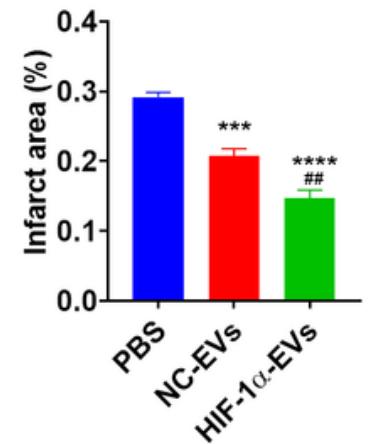

G
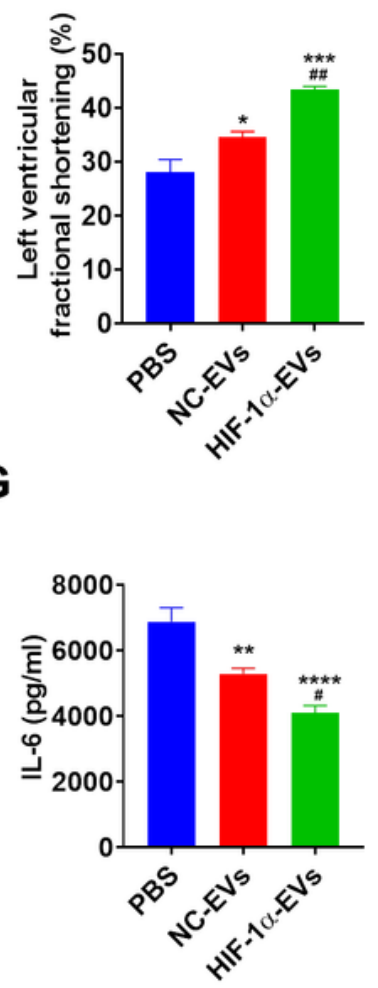

\section{Figure 4}

HIF-1a-EVs effectively preserved cardiac function in a rat MI model. (A) Dil-labeled exosomes were injected into the infarcted heart of rats for $6 \mathrm{~h}(50 \mu \mathrm{g}$ exosomes per rat). Representative images of post-

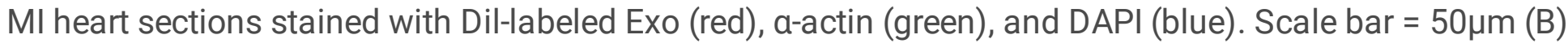
Representative echocardiogram of rat heart in different groups at 28 days post-MI. (C) HE staining and Masson Trichrome staining at the border zone on Day 28 after MI. (D and E) Significantly enhanced LVEF 
and LVFS in rats transplanted with MSC-EVs and HIF-1a-EVs compared with control groups. (F)

Quantitative data for the fibrotic area. Continuous variables were described by means \pm SEM. $n=5-6$ for each group. ${ }^{*} \mathrm{P}<0.05,{ }^{*} \mathrm{P}<0.01,{ }^{\star *} \mathrm{P}<0.001,{ }^{*} * \star \mathrm{P}<0.0001$ vs. $\mathrm{PBS}$ group; $\# \mathrm{P}<0.05, \# \# \mathrm{P}<0.01$ vs. NCEVs group.

A

\section{PBS}

NC-EVs

HIF-1 $\alpha-E V s$

C

PBS

NC-EVs

HIF-1 $\alpha-E V s$

$\mathbf{F}$
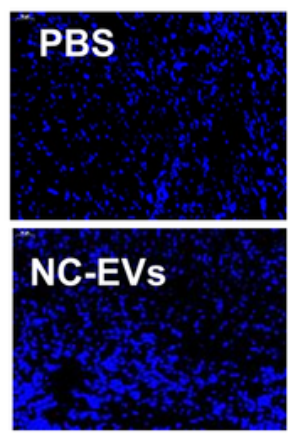

HIF-1 $\alpha-E V s$
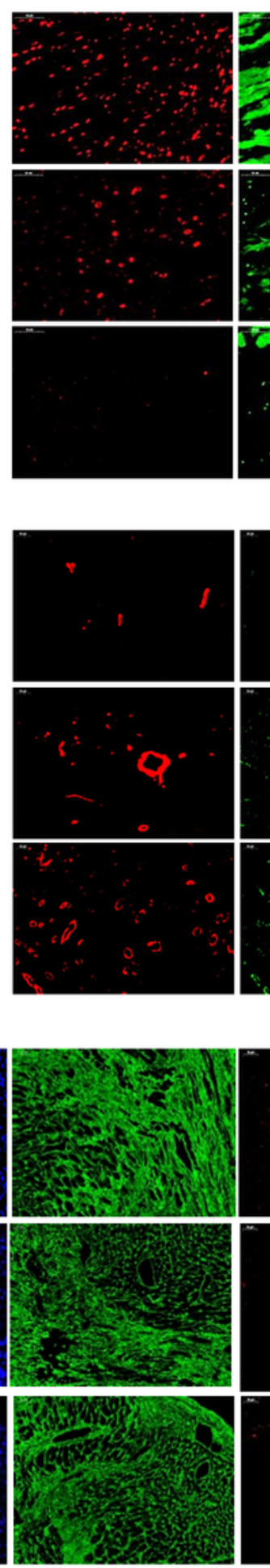
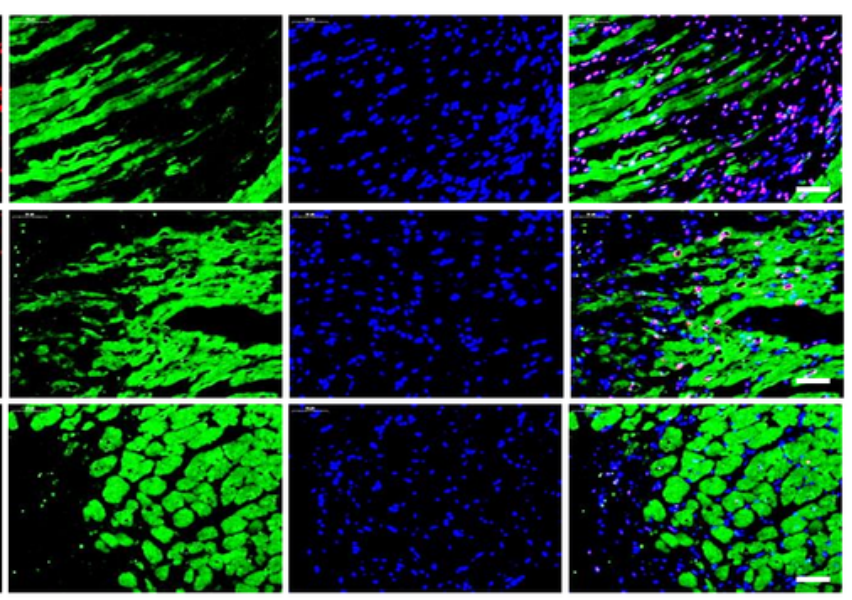

\section{B}
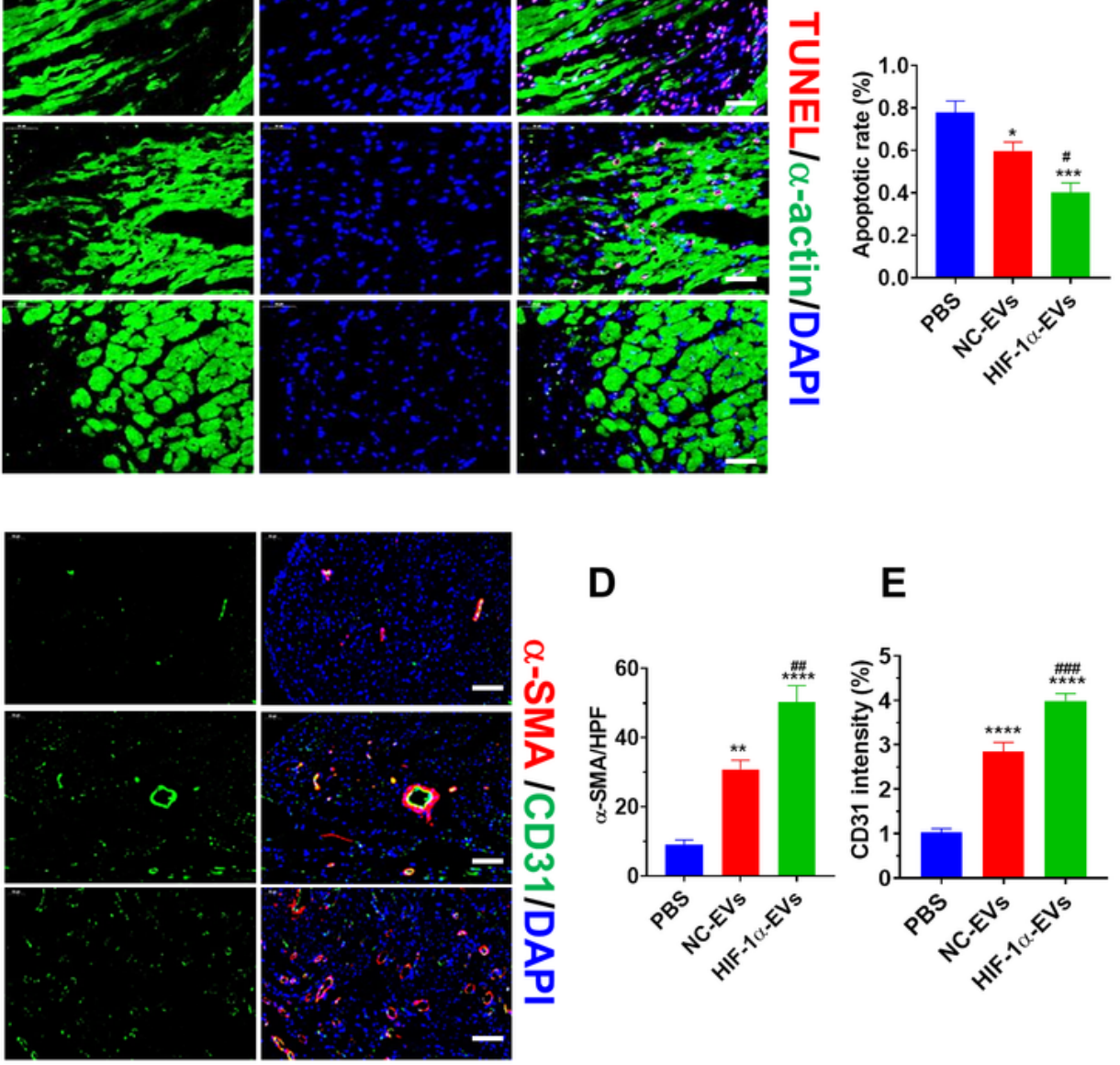

\section{Boder Zone Infarct area \\ Infarct area}

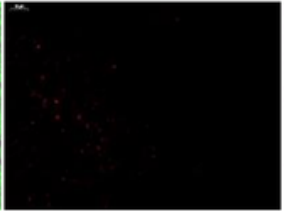

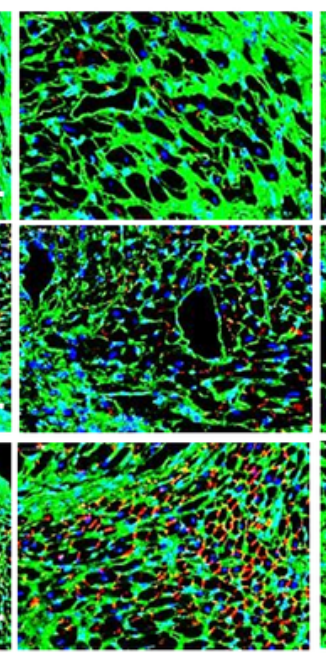

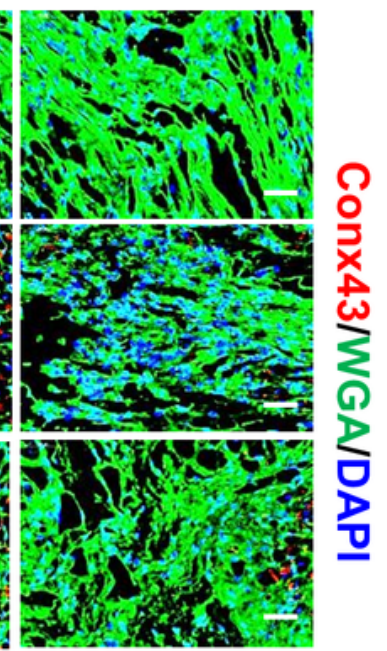

Figure 5 
HIF-1a-EVs inhibited apoptosis and promoted angiogenesis in a rat MI model. (A)TUNEL staining at the border zone on Day 28 post-MI with TUNEL-positive cells. Red, TUNEL-positive nuclei; Green, a-actin; blue, DAPI-stained nuclei. Scale bar $=50 \mu \mathrm{m}$ (B) Quantification of TUNEL-positive cells in each group. (C) Neovascularization at the border zone on Day 28 post-MI was identified by staining with a-SMA (red) and CD31 (green) and nuclei (blue). Scale bar $=50 \mu \mathrm{m}$ (D and E) Quantification of a-SMA-positive cells, CD31positive cells among different groups. (F) Border zone sections obtained 4 weeks after MI were immunofluorescently stained for Con43 expression. Red, Conx43; Green, a-actin; blue, DAPI-stained nuclei. Continuous variables were described by means \pm SEM. $n=5-6$ for each group. ${ }^{*} P<0.05, * * P<0.01$, ${ }^{\star * \star} P<0.001,{ }^{* \star \star \star} P<0.0001$ vs. PBS group; $\# P<0.05, \# \# P<0.01$ vs. NC-EVs group. 


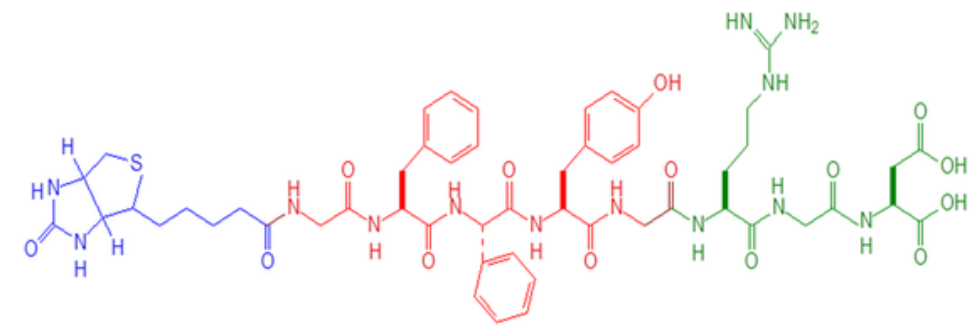

Biotin

-GFFYG

D

RGD

C

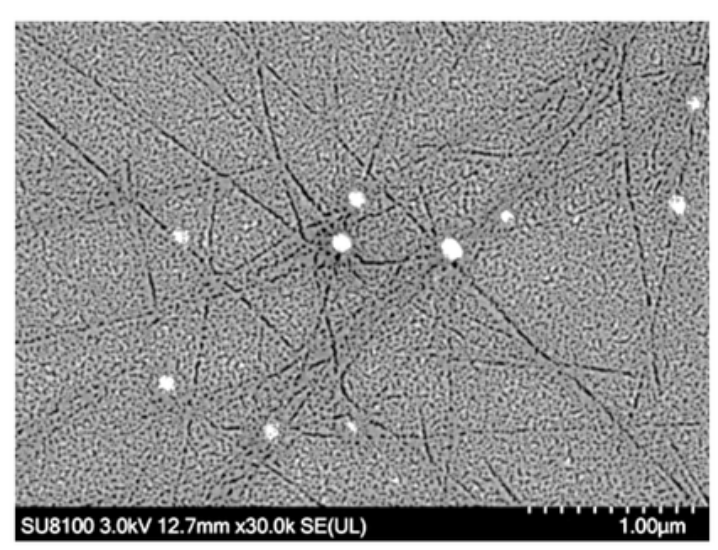

EVs supermolecular nanofibers containing RGD peptides

AMI Model
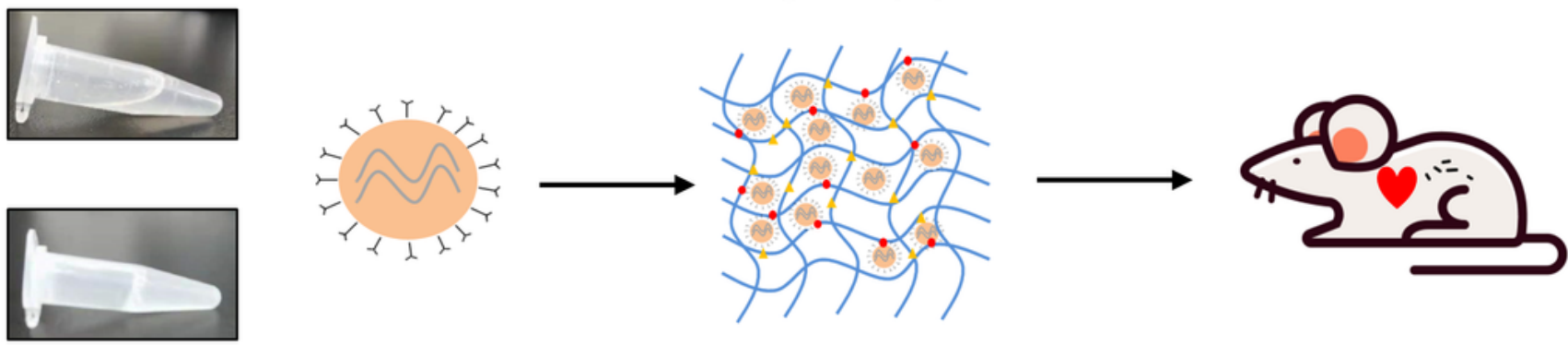

E

Day 1

Day 2

Day 3

Day 5

Day 7

Day 14

HIF-1 $\alpha-E V s$

RGD-Biotin
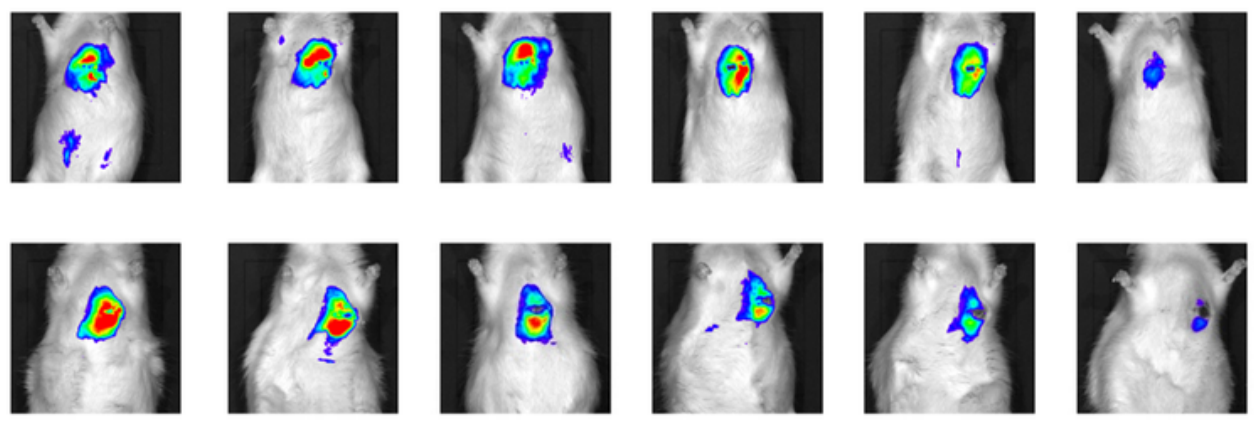

NC-EVs

RGD-Biotin
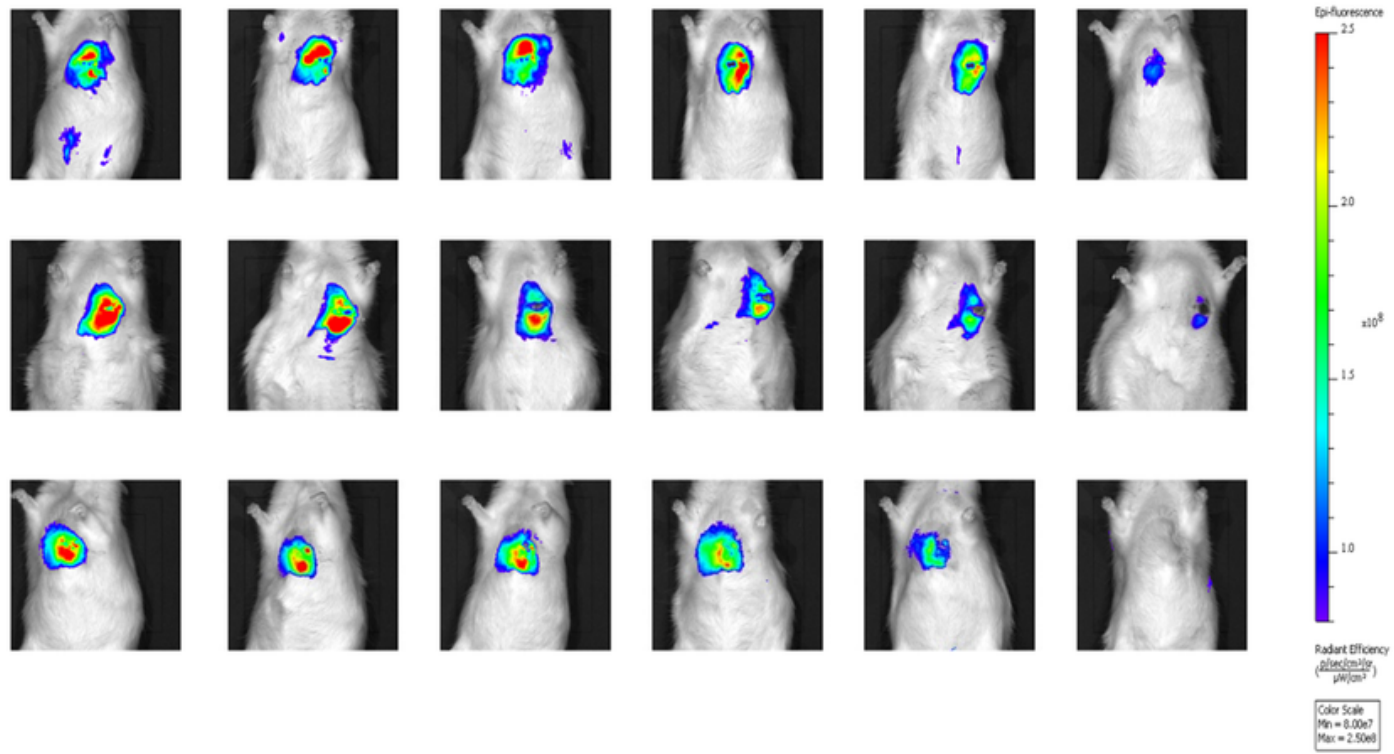

Figure 6

EVs/RGD-biotin hydrogels enhanced the retention and stability of HIF-1a-EVs. (A) Chemical structure of the RGD-biotin molecule. (B) SEM image of self-assembling RGD-biotin hydrogel revealed the formation of entangled nanofibers formed by the RGD-biotin molecule. (C) Biotin-GFFYGRGD was dissolved in PBS. After that, the suspension was heated to about $95^{\circ} \mathrm{C}$ in order to dissolve the compound completely, and hydrogels formed after cooling back to room temperature. (D) EVs were incorporated in Biotin-GFFYGRGD 
and then injected into infarcted rat hearts. (E) The fate of EVs in vivo was tracked by bioluminescence imaging. Representative images are from rats receiving EVs incorporated in RGD hydrogels (EV/RGDbiotin) and EVs without scaffolds (EVs). Continuous variables and categorical variables were described by means \pm SEM and percentages. $n=3$ for each group.

A

HIF-1 $\alpha-E V s$
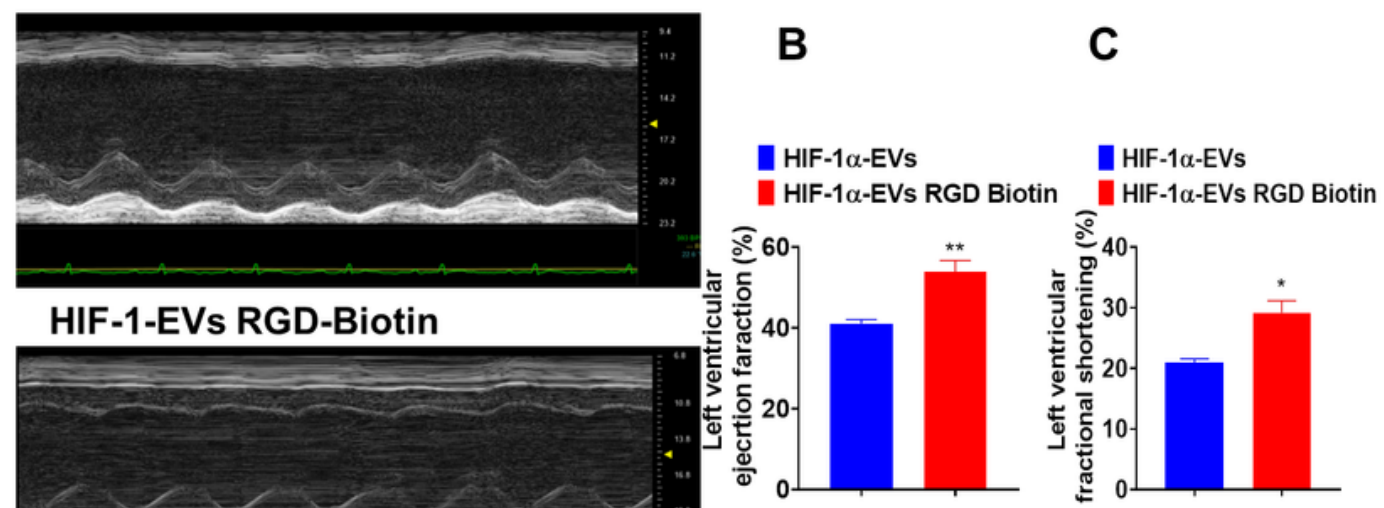

D

HIF-1-EVs RGD-Biotin

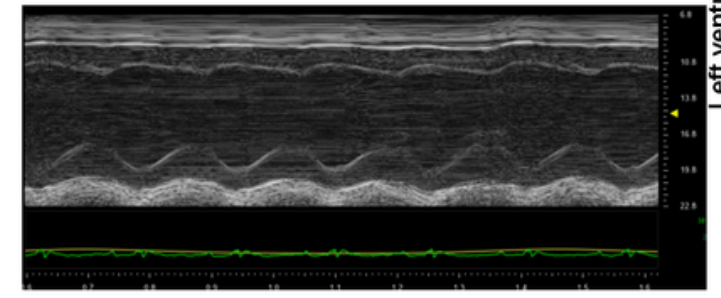

E
HIF-1 $\alpha-E V s$
HIF-1 $\alpha$-EVs
RGD-Biotin
HIF-1 $\alpha-E V s$
HIF- $1 \alpha-E V s$
RGD-Biotin

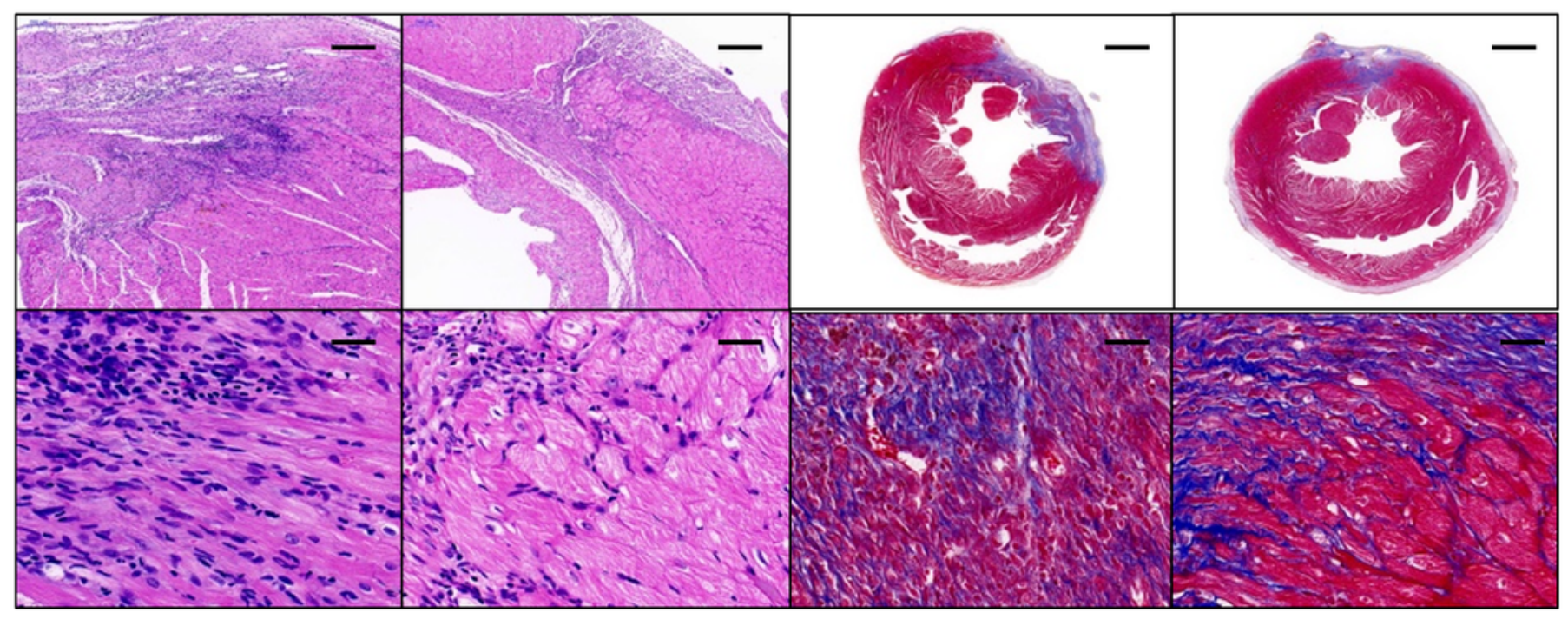

Figure 7 
EVs/RGD-biotin hydrogels improved Cardiac function after AMI. (A) Representative echocardiogram of rat heart between HIF-1a-EVs group and HIF-1a-EVs RGD-Biotin group at 28 days post-MI. (B and C)

Significantly enhanced LVEF and LVFS in rats transplanted with MSC-EVs and HIF-1a-EVs compared with control groups. (D) HE staining and Masson Trichrome staining at the border zone on Day 28 after MI. (E) Quantitative data for the fibrotic area. Continuous variables were described by means \pm SEM. $n=4-6$ for each group. ${ }^{*} P<0.05,{ }^{*} P<0.01$.

A

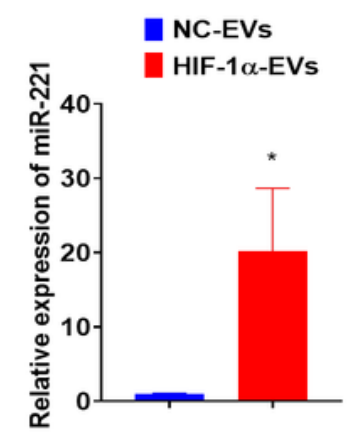

B
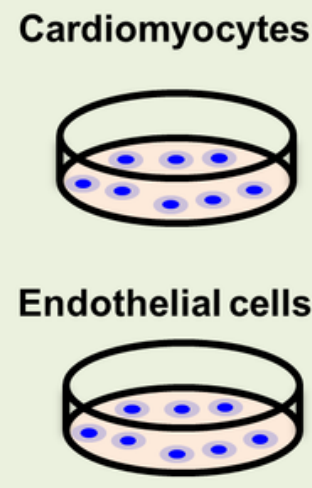
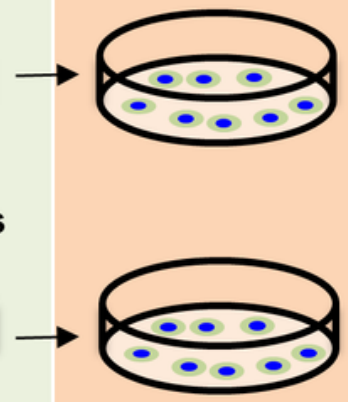

H/SD

Treated with

HIF-1 $\alpha$-EV-InhibitormiR221

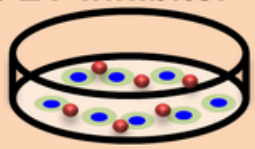

Treated with

HIF-1 $\alpha$-EV-Inhibitor ${ }^{N C}$

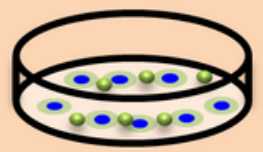

C

HIF-1 $\alpha-E V-I n h i b i t o r^{N C}$

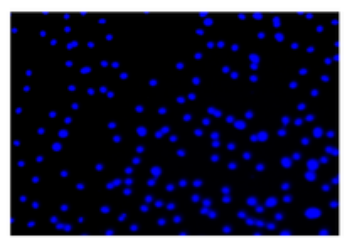

HIF-1 $\alpha$-EV-InhibitormiR-221
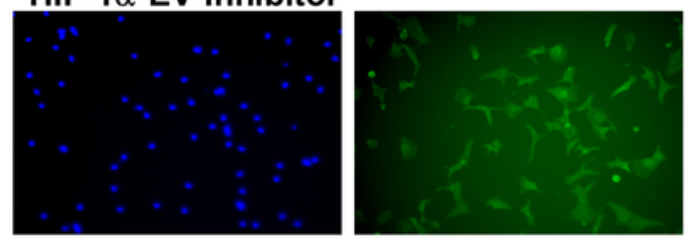

E

HIF-1 $\alpha-E V-I n h i b i t o r^{N C}$
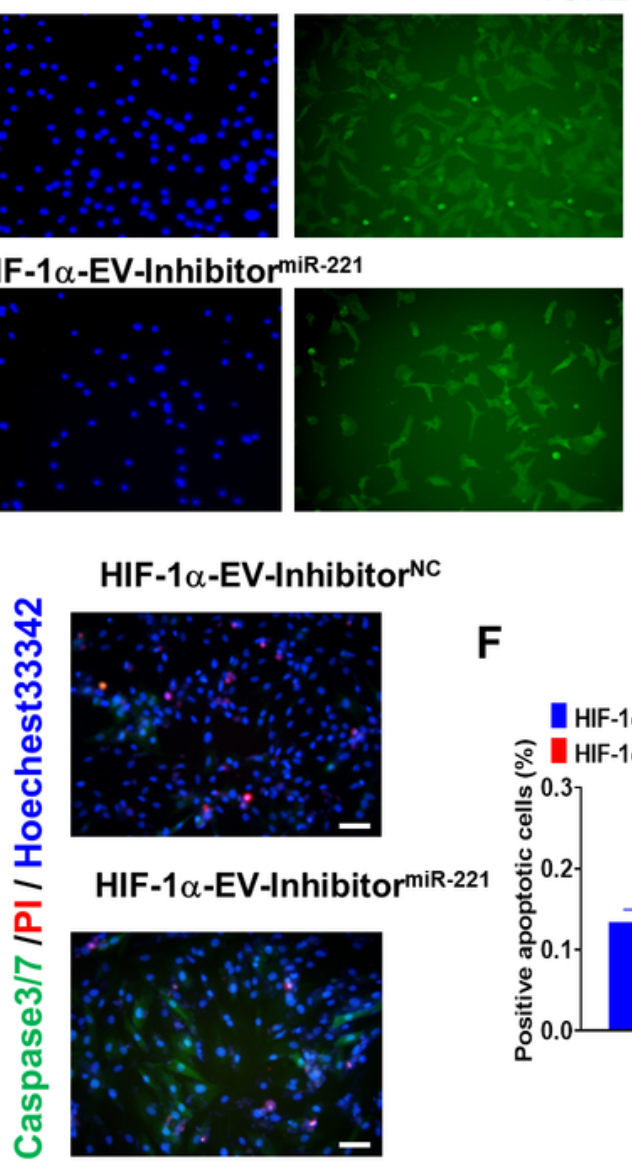

$\mathbf{F}$ ఏ 0.3
TUNEL / $\alpha$-actin/ DAPI
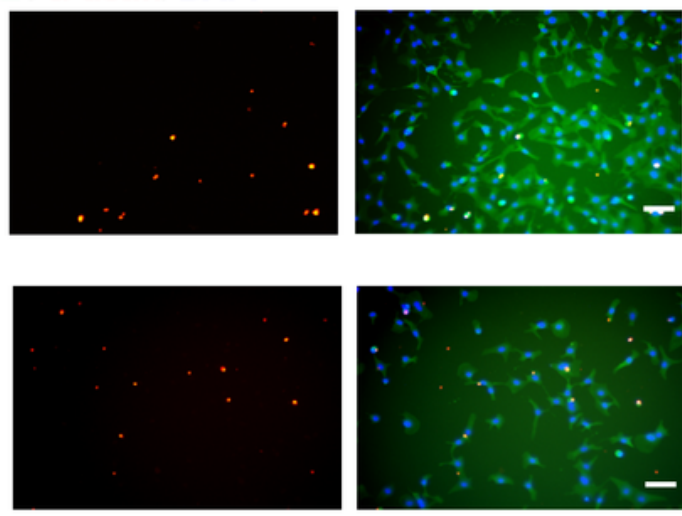

G

HIF-1 $\alpha$-EV-Inhibitor ${ }^{\text {NC }}$ ○ HIF-1 $\alpha$-EV-Inhibitor ${ }^{\text {miR-221 }}$

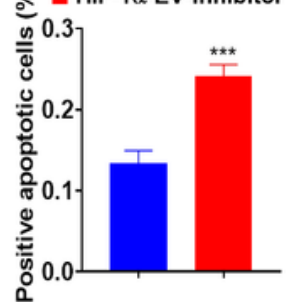

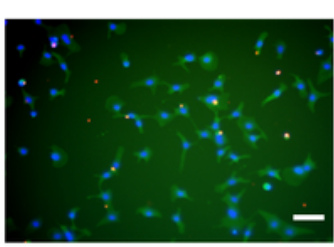

HIF-1 $\alpha-E V-I n h i b i t o{ }^{N C}$

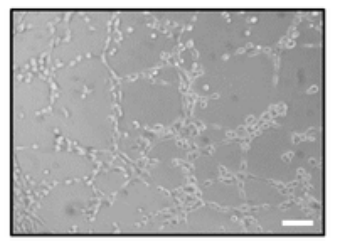

HIF-1 $\alpha-E V-I n h i b i t o r^{m i R-221}$

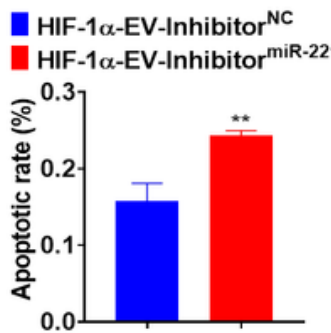

H

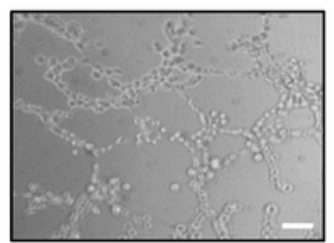

D 
Inhibition of miR-221-3p Reversed the Protective Effects of EVs on Apoptosis and Angiogenesis. (A) Expression values for miR-221-3p in MSC-EVs and HIF-1 $a-E V s$ by qRT-PCR. (B) Experimental design. To inhibit miR-221-3p in HIF-1a-EVs. HIF-1a-EVs-inhibitormiR-221 and HIF-1a-EVs-inhibitorNC were incubated with cardiomyocytes or HUVECs under H/SD condition. (C and D) TUNEL staining at the border zone 4 weeks after $\mathrm{MI}$, and quantification analysis of percentage of apoptotic cells between two groups. Scale bar $=100 \mu \mathrm{m}(\mathrm{E}-\mathrm{F})$ Caspase3/caspase7 activity and quantification analysis in cardiomyocytes treated with HIF-1a-EVs-inhibitormiR-221 and HIF-1a-EVs-inhibitorNC under H/SD condition. Scale bar $=100 \mu \mathrm{m}$ (G) Representative images showing tube formation in HUVECs treated with HIF-1a-EVs-inhibitormiR-221 and HIF-1a-EVs-inhibitorNC. Scale bar $=100 \mu \mathrm{m}(\mathrm{H})$ Quantification of tube length in each group. Continuous variables and categorical variables were described by means \pm SEM and percentages. $n=6$ for each group. ${ }^{*} \mathrm{P}<0.05$; ${ }^{\star \star} \mathrm{P}<0.01 ;{ }^{* \star *} \mathrm{P}<0.001$. 


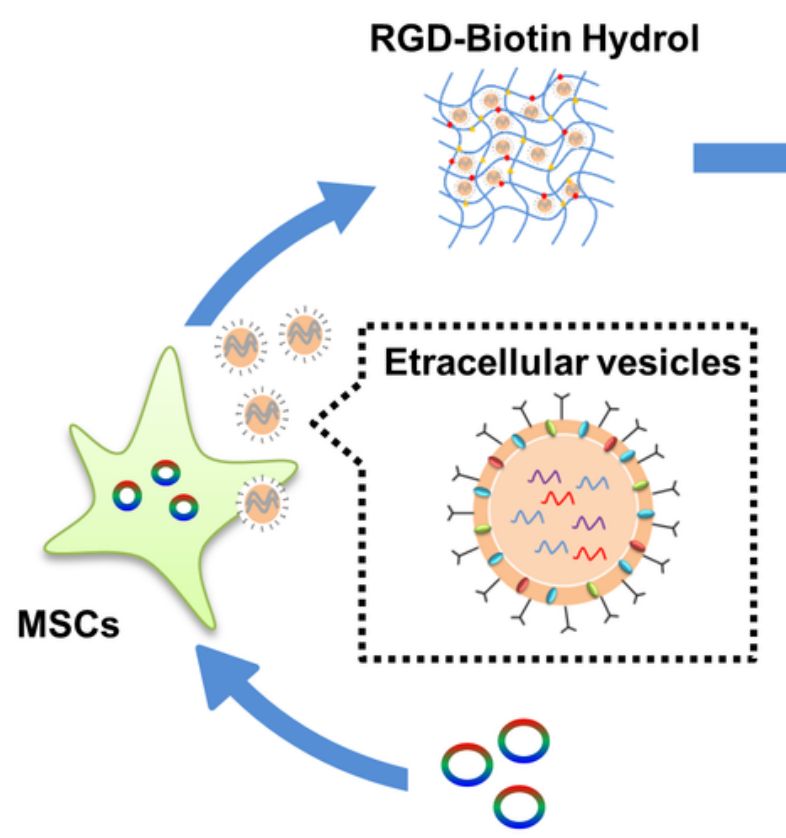

HIF- $1 \alpha$ lentivirus
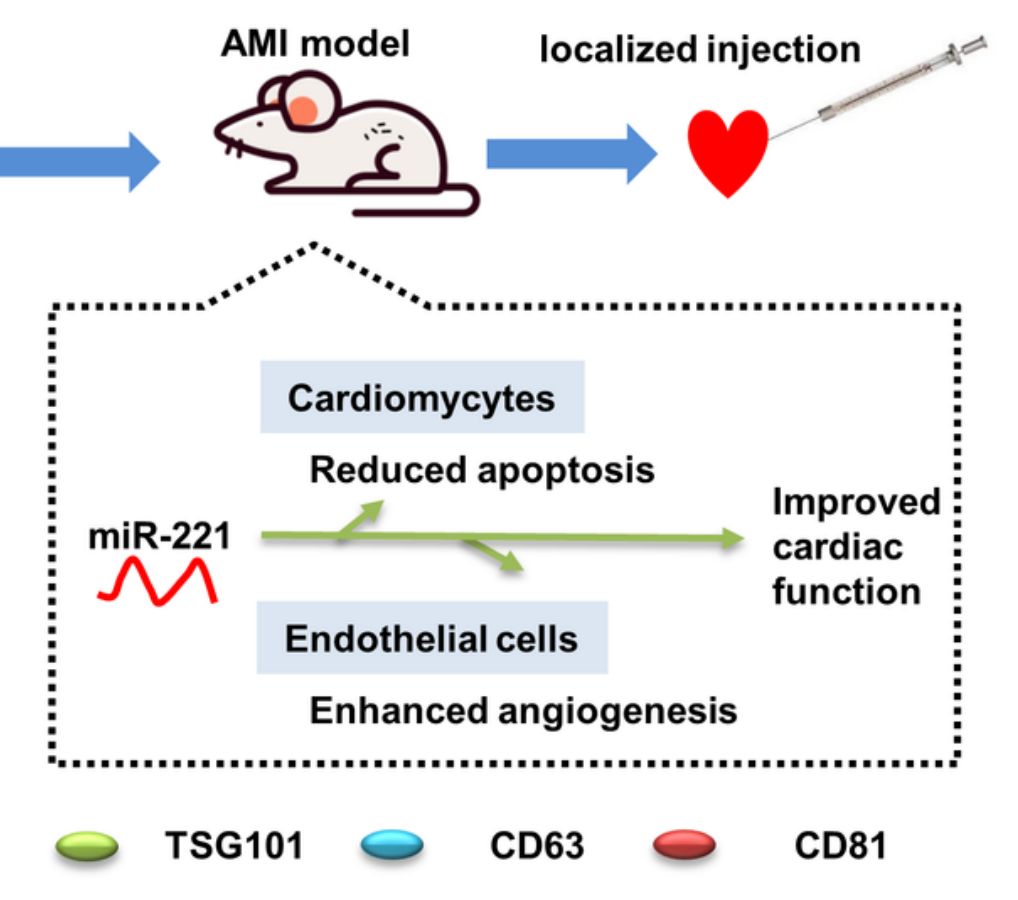

Figure 9

Schematic of the working hypothesis. EVs released by MSCs with stabilized overexpression of HIF-1a contribute to angiogenesis and anti-apoptosis in part via enhanced expression of miR-221-3p. RGD hydrogels increased the therapeutic efficacy of HIF-1a engineered MSC-derived EVs at a functional, histopathological and molecular level. 


\section{Supplementary Files}

This is a list of supplementary files associated with this preprint. Click to download.

- 2021722suppHIFv2.docx 